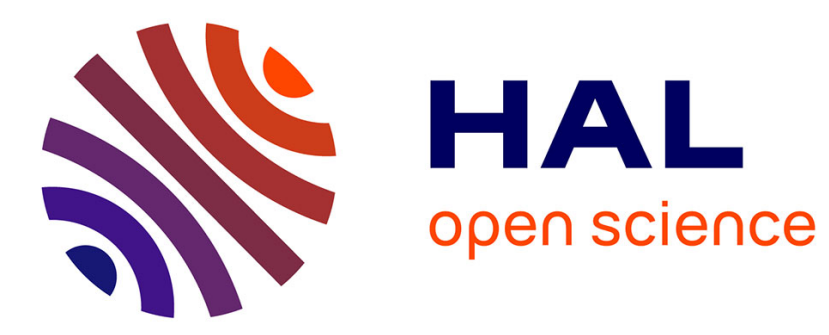

\title{
Mean steady granular force on a wall overflowed by free-surface gravity-driven dense flows
}

\author{
Thierry Faug, R. Beguin, B. Chanut
}

\section{To cite this version:}

Thierry Faug, R. Beguin, B. Chanut. Mean steady granular force on a wall overflowed by free-surface gravity-driven dense flows. Physical Review E : Statistical, Nonlinear, and Soft Matter Physics, 2009, 80 (2), 13 p. 10.1103/PhysRevE.80.021305 . hal-00455548

\section{HAL Id: hal-00455548 \\ https://hal.science/hal-00455548}

Submitted on 10 Feb 2010

HAL is a multi-disciplinary open access archive for the deposit and dissemination of scientific research documents, whether they are published or not. The documents may come from teaching and research institutions in France or abroad, or from public or private research centers.
L'archive ouverte pluridisciplinaire HAL, est destinée au dépôt et à la diffusion de documents scientifiques de niveau recherche, publiés ou non, émanant des établissements d'enseignement et de recherche français ou étrangers, des laboratoires publics ou privés. 


\title{
Mean steady granular force on a wall overflowed by free-surface gravity-driven dense flows
}

\author{
Thierry Faug, Rémi Beguin, Benoit Chanut* \\ Cemagref, ETGR, 38402 St-Martin d'Hères, France
}

\begin{abstract}
We studied free-surface gravity-driven recirculating flows of cohesionless granular materials down a rough inclined plane and overflowing a wall normal to the incoming flow and to the bottom. We performed 2D spherical particle discrete element simulations using a linear damped spring law between particles with a Coulomb failure criterion. High-frequency force fluctuations were observed. This paper focuses on the mean steady force exerted by the flow on the obstacle versus the macroscopic inertial number of the incoming flow, where the inertial number measures the ratio between a macroscopic deformation timescale and an inertial timescale. A triangular stagnant zone is formed upstream of the obstacle and sharply increases the mean force at low incoming inertial numbers. A simple hydrodynamic model based on depth-averaged momentum conservation is proposed. This analytical model predicts the numerical data fairly well and allows us to quantify the different contributions to the mean force on the wall. Beyond this model, our study provides an example of the ability of simple hydrodynamic approaches to describe the macroscopic behavior of an assembly of discrete particles, not only in terms of kinematics, but also in terms of forces.
\end{abstract}

PACS numbers:

Keywords:

*Electronic address: thierry.faug@cemagref .fr

1 (August 7, 2009) 


\section{INTRODUCTION}

The importance of granular materials in geophysics and in various industrial processes has resulted in extended research on granular flows at the frontier between physics, soil mechanics and fluid mechanics. Flows around obstacles and the force those granular flows are able to exert on the obstacle are important issues when applied to storage and conveying bulk solids [1] and also in geophysical flows [2-4]. Granular drag on objects was approached by the pioneering work of Wieghardt [5, 6], who provided an early systematic experimental and analytical study of granular flows around immersed objects. In those free-surface flows, he showed the drag force depended relatively little on velocity and he observed a pile-up in front of the immersed object as well as depression of the surface in the wake. A recent experimental study on dense granular flows around an immersed cylinder in a vertical chute [7] showed that the mean drag is independent of the mean upstream velocity. The subject also contributed experiments on two-dimensional flows in a vertical bin around various inserts [1]. The authors recorded velocity contours and observed stagnant zones in front of the inserts.

Many recent studies have focused on the drag force on small obstacles such as cylinders in the case of rapid - dilute - granular flows [8, 9], including interstitial gas effects [10, 11]. In the dilute regime, the force is proportional to the square of the incoming velocity as predicted from kinetic theory for granular gas. The importance of shock waves formed in front of the obstacle has been shown for this rapid regime $[12,13]$ and has been the subject of valuable recent studies [18-24].

The quasic-static regime has also been investigated by Wieghardt $[5,6]$. A series of recent studies examined drag forces on immersed obstacles in a rotating granular bed at very low velocities [25-27]. Experimental measurements showed that the drag force is linearly

dependent on the cylinder diameter, quadratically dependent on the depth of insertion and independent of velocity [25]. The effect of obstacle shape on drag force and its influence on jamming behavior has been analyzed [27] as well as stick-slip fluctuations in granular drag [26]. All these studies refer to horizontal motion and showed that the average pressure is scaled as the immersion depth. A recent study investigated the average drag forces for obstacles slowly plunging into and withdrawing from shallow beds of different granular materials, and showed that the average pressure is scaled as the immersion depth exhibiting 
a power law behaviour rather than a linear behavior [28].

Since the studies of Wieghardt [5] and Tuzun and Nedderman [1], little has been studied on the drag of dense granular flows and the effects of the stagnant zone observed in front of the obstacle. In the present paper, dense granular flows will systematically refer to assemblies of grains moving at higher velocity than the grains in the quasi-static regime mentioned just above but at lower velocity than the grains in the rapid dilute regime. This regime refers to the so-called granular liquid regime mentioned in [29]. A typically sized roughness close to the diameter of the flowing grains is a condition to obtain this type of dense regime. In the dense granular regime, when the obstacle has a typical size close to the flow depth, large stagnant zones or dead zones are able to form in front of the obstruction, while grains that are non trapped inside the dead zone continue to flow around the obstacle. These stagnant zones observed in the dense regime are not accompanied by a granular jump (large discontinuity in flow depth and velocity), as described earlier for rapid flows on smooth beds [14-17]. Furthermore, free-surface curvatures and large flow-depth gradients are observed in the vicinity of the obstacle. All these conditions result in a mean force on the obstacle that cannot be expressed as (i) a simple function of the incoming depth-averaged velocity of the flow, as is usually observed for granular flows around small immersed obstacles in the dilute regime $[8,9,12,13]$ or (ii) a simple function of a depth of insertion (equal to the flow depth when the small object is located at the base of the flow) in the quasi-static regime $[25,27,28,30]$.

This paper focuses on $2 D$ free surface gravity-driven flows of dense granular materials down an inclined plane and overflowing a wall normal to the bottom. These flows correspond to a flow geometry typically encountered in geophysical flows when avalanches overtop protection dams [4]. A roughly triangular dead zone, whose length greatly depends on the slope inclination, is formed upstream of the wall and largely influences the mean force on the wall. We investigated this mean force using discrete numerical simulations combined with a hydrodynamic modeling approach. The numerical simulations method and results are presented first. Then we describe a simple hydrodynamic analytical model showing that the mean force is the sum of many contributions: the weight of the granular material (motionless grains inside the dead zone and moving grains above), the incoming momentum force, the incident pressure force and the basal friction force inside the dead zone. Based on simple arguments to calibrate the free parameters, the predictions from the proposed 
analytical model are successfully compared to numerical results.

\section{DISCRETE NUMERICAL SIMULATIONS}

\section{1. $\quad$ Numerical methods}

\section{Simulation method and contact law}

Numerical simulations are carried out using the molecular dynamics method, as introduced by Cundall [31] and largely used to simulate dense granular flows (see for example [32-34]). We defined the normal contact force as the sum of two contributions, an elastic one $N^{e}$ (spring) and a viscous one $N^{v}$ (dashpot). In the simplest case used here, the normal elastic force $N_{i j}^{e}$ is assumed to depend linearly on the overlap $\delta_{i j}$ (the displacement of the spring): $N_{i j}^{e}=k_{n} \delta_{i j}$, where $k_{n}$ is the normal stiffness. The dashpot contributes a normal dissipative force $N^{v}$ proportional to the time derivative of the overlap: $N_{i j}^{v}=C_{i j} \dot{\delta}_{i j}$, where $C_{i j}$ is a normal damping coefficient that may be related to a coefficient restitution $e$ in a binary collision of cohesionless grains $[35,36]$. The sum of both contributions $N_{i j}$ is restricted to being repulsive, i.e., tensile normal forces are not allowed, as the particles are assumed to be non cohesive. Finally, the normal contact force may be expressed as follows: $N_{i j}=k_{n} \delta_{i j}+C_{i j} \dot{\delta}_{i j}$. Two microscopic parameters are then needed: the normal stiffness $k_{n}$ and the damping coefficient $C_{i j}$ (or restitution coefficient $e$ ) to define the normal intergranular force. The tangential component $T_{i j}$ of the contact force is implemented in terms of a linear spring $T_{i j}=k_{t} u_{i j}$, where $k_{t}$ is the tangential stiffness and $u_{i j}$ the displacement of the spring. $T_{i j}$ is restricted to absolute values smaller than $\mu N_{i j}^{e}$ according to friction between grains described by a Coulomb condition enforced with the sole elastic part of the normal force. Here the local particle friction parameter $\mu$ is introduced. When this threshold is reached, the tangential relative motion is regarded as sliding with the sliding friction $\mu N_{i j}^{e}$ (directed opposite the tangential relative velocity). Finally the tangential contact force may be expressed as follows: $T_{i j}=\min \left(\mu N_{i j}^{e}, k_{t} u_{i j}\right)$. Two miscroscopic parameters are then needed: the tangential stiffness $k_{t}$ and the local particle friction parameter $\mu$ to define the tangential intergranular force. 


\section{Microscopic parameters}

The simulated system is an assembly of spheres of average diameter $d$ and density $\rho_{P}$ (average mass $\left.m=1 / 6 \rho_{P} \pi d^{3}\right)$. A small polydispersity ( $\pm 10 \%$ in size) is introduced to prevent crystallization. The average particle diameter is $d=1 \mathrm{~mm}$ and the particle density is $\rho_{P}=2450 \mathrm{~kg} \mathrm{~m}^{-3}$ corresponding to the glass material. The normal stiffness $k_{n}$ is calculated from the Young modulus $E$ [37]: $k_{n} \approx E d$. The Young modulus of the glass material is $E=69 G P a$, which gives $k_{n}=6.910^{7} \mathrm{~N} \mathrm{~m}^{-1}$ but $k_{n}$ was reduced to $k_{n}=10^{4} \mathrm{~N} \mathrm{~m}^{-1}$ with respect to the limit of rigid grains (no influence of the stiffness above this limit), in order to decrease the calculation time.

It has been previously shown that the dimensionless number $N_{1}=k_{n} /\left(\rho d^{3} \dot{\gamma}^{2}\right.$ ) (where $\dot{\gamma}$ is the shear rate and $\rho$ the density) has to be greater than $10^{4}$ in order to conclude that we are in the limit where grains behave as if they are perfectly stiff [36]. $N_{1}$ can be thought as the inverse of the square of the ratio between the shear velocity $u_{\dot{\gamma}}=\dot{\gamma} d$ and the sound velocity $c_{s}=\sqrt{k /(\rho d)}[36]$. The condition $N_{1}>10^{4}$ gives $u_{\dot{\gamma}} / c_{s}<10^{-2}$ (condition 1). This condition 1 controls the overlap generated by flow inertia and gives the following condition on the shear rate (with $k_{n}=10^{4} \mathrm{~N} \mathrm{~m}^{-1}$ and a density $\rho=\phi \rho_{P}$ where $\phi=0.55$ is a typical volume fraction): $\dot{\gamma}<860 \mathrm{~s}^{-1}$. The dense granular flows investigated in this paper occur below this limiting value of the shear rate (as well as many similar experimental granular flows [38]). We have also to consider the overlap generated by quasistatic mechanisms such as the compression of force chains. Similarly to the number $N_{1}$, we can define the dimensionless number $N_{2}=k_{n} /(P d)$ for spherical grains, which can be interpreted as the inverse of the square of the ratio between the characteristic velocity of grains rearrangement $u_{P}=\sqrt{P / \rho}$ (where $P$ is the pressure) and the sound velocity $c_{s}$. By analogy to the condition 1 , the condition $N_{2}>10^{4}$, which gives $u_{P} / c_{s}<10^{-2}$, has to be respected (condition 2). For dense granular flows, the typical pressure level is maximum at the bottom and may be approximated by the hydrostatic pressure $P \approx \rho g h$. The condition 2 gives the following condition on the flow depth (with $k_{n}=10^{4} \mathrm{~N} \mathrm{~m}^{-1}$ ): $h<75 d$. The granular flows investigated in the paper are below this limiting value of the flow depth (thereafter, in Fig. 1b, are provided the typical values of the flow depth). Furthermore, recent discrete simulations on dense granular flows showed that the effect of variations in $k_{n}$ is minimal as long as $k_{n}>2.10^{5} \mathrm{mg} / d$ [39]. With our values for $d$ and $m$, it gives $k_{n}>2570$ 
$\mathrm{N} \mathrm{m}^{-1}$, which is also compatible with the chosen value for $k_{n}$.

In the rigid grains limit, it has been shown that the macroscale behavior does not depend on $\mu$ (except for frictionless grains $\mu=0$ ) and e (except for the extreme values $e=0$ and $e=1$ ) for plane shear flows without gravity [34]. It has also been shown that the flow behavior (bulk density and velocity profile) depends little on the local particle friction if $\mu>0.5$ for steady flows down a rough inclined plane (see Fig. 9 in [32]). In our simulations, we used $\mu=0.5$ corresponding to a typical value for glass beads. The restitution coefficient $e$ was also shown to only slighly influence the bulk density and the velocity profile of steady dense granular flows down rough inclined planes if $e<0.9$ (see figure 10 in [32]). In the dense granular regime, the macroscopic flow quantities are known to be almost insensitive to the coefficient of restitution $e[29,40]$. In our simulations, we chose $e=0.5$. It has also been shown that the $k_{t} / k_{n}$ ratio has a very small influence $[32,36]$. Here we used $k_{t} / k_{n}=1 / 2$, which gives $k_{t}=510^{3} \mathrm{~N} \mathrm{~m}^{-1}$. The commercial Particle Flow Code was used here $\left(\mathrm{PFC}^{2 D}\right.$ version 3.0 [41]). In our simulations, the time step was kept constant at $410^{-6} s$, which guaranteed the stability of our calculations.

\section{Flow geometry}

We defined a channel of length $L_{c}=1700 d$ and a reservoir of length $L_{r}=150 d$. The roughness of the bottom was made with grains of a mean diameter $d=1 \mathrm{~mm}$ with the same properties as the grains in motion. The channel was constantly fed by releasing grains into the reservoir. Grains moved out of the reservoir by an aperture of constant height $H_{r}=35 d$ (see inset in Fig. 1a). After a certain simulation time, the imposed height $H_{r}$ at the exit of the channel led to a constant mass flow rate. We investigated two types of flow in a large range of slope inclinations $\left(16^{\circ} \leq \theta \leq 32^{\circ}\right)$ : (i) flows with no obstacles in order to characterize our flows regarding the existing literature reporting dense granular flows down rough inclined planes and (ii) flows overflowing a vertical wall normal to the bottom and located at a distance $900 \mathrm{~d}$ from the exit of the reservoir. The following section presents the results. 


\section{2. $\quad$ Numerical results}

Control flows without an obstacle

Dense granular flows down rough inclined planes have been thoroughly studied and an overview of recent progress and remaining unanswered questions can be found in [29, 40]. This section reports a summary of the main results obtained from our discrete simulations of control flows with no obstacles. We show that these results are compatible with previous reports in the literature [40]. The existence of steady and uniform flows in a large range of slope inclinations $\left[\theta_{\min }, \theta_{\max }\right]$ has been shown for dense granular flows down a rough inclined plane $[32,40,42]$. The function $h_{\text {stop }}(\theta)$ has been defined as the thickness of the granular material left by a steady and uniform flow at slope inclination $\theta$ and can be measured. The angle $\theta_{\text {max }}$ is the angle for which no grains are able to stay on the inclined plane $\left(h_{\text {stop }}=0\right)$ and the angle $\theta_{\text {min }}$ is the angle for which $h_{\text {stop }}$ tends towards $\infty$ (no flow). We determined the function $h_{\text {stop }}(\theta)$ numerically, which is depicted in Fig. 1a. The curve can be fitted by the function $h_{\text {stop }}(\theta)=B d\left[\left(\theta_{\max }-\theta\right) /\left(\theta-\theta_{\text {min }}\right)\right]$ where $B$ is a constant depending on material properties [40]. We found the following values: $\theta_{\min } \approx 14^{\circ}, \theta_{\max } \approx 24^{\circ}$ and $B=2.3$. It is found to be in agreement with previous data on 2D flows [32, 40, 43].

A single dimensionless number called the inertial number has been defined and may be interpreted as the ratio [40] between (i) a microscopic time scale $d / \sqrt{P / \rho_{P}}$, which represents the time it takes for a particle of density $\rho_{P}$ to fall in a hole of size $d$ under the pressure $P$ giving the typical time scale of rearrangements, and (ii) a macroscopic time scale $1 / \dot{\gamma}$ linked to the mean deformation ( $\dot{\gamma}$ is the local shear rate). $I$ is the square root of the Savage number [44] or the Coulomb number $[45,46]$ previously introduced in the literature. The averaged inertial number $I$ may be estimated from depth-averaged velocity and thickness of the flows assuming a Bagnold-like velocity profile [40]:

$$
I=\frac{5 \bar{u} d}{h^{3 / 2} \sqrt{2 g \cos \theta}}
$$

Fig. 1b shows the velocity profiles obtained at different slope inclinations and at the position $x / d=900$. The Bagnold profiles (shown in dash lines in Fig. 1b) roughly fit the numerical data, except at the base of the flow as it has been previously reported [40]. Fig. 1c shows the macroscopic inertial number $I$, calculated from Eq. (1), along the distance $x$ from 
the reservoir. For steady and uniform flows, we can define a unique value of $I$ far enough from the reservoir. Above $\theta_{\max }$, we observed a continuous increase in the inertial number $I$ along $x$ showing that the flows are non-uniform flows. For these gradually accelerated flows, we defined the inertial number $I$ at two positions: $x / d=900$, corresponding to the location of the wall in the second set of simulations, and $x / d=1500$ (maximum position up to which we took measurements because for $x / d>1500$ the flow properties were influenced by the boundary limit due to the end of the inclined plane at $x / d=1700)$.

The robust scaling has been shown for the variation of the basal friction coefficient $\mu^{*}$ versus the inertial number $I$ for plane shear flows [34]:

$$
\mu^{*}=\mu_{\min }^{*}+b I
$$

where $\mu_{\text {min }}^{*}=\tan \theta_{\min }$ and $b$ is a constant. Another scaling is proposed in the literature for $\mu^{*}(I)$ for inclined planes $[47,48]$ :

$$
\mu^{*}=\mu_{\min }^{*}+\frac{\Delta \mu}{I_{0} / I+1}
$$

where $\Delta \mu=\tan \theta_{\max }-\mu_{\min }^{*}$ and $I_{0}$ is a constant typically equal to 0.3 for glass beads.

The depth-averaged equations, introduced by [49] and recently revisited $[29,50]$ in the context of shallow granular flows down an inclined plane, allow us to estimate the effective friction coefficient $\mu^{*}$. The acceleration is balanced by the gravity parallel to the plane, the tangential stress between the fixed bottom and the flowing layer, and a pressure force related to the thickness gradient $[29,49,50]$. The momentum balance is reduced to the following equation in steady 2D flow conditions:

$$
\beta \frac{\partial h \bar{u}^{2}}{\partial x}=\left(\tan \theta-\mu^{*}-k \frac{\partial h}{\partial x}\right) g h \cos \theta .
$$

The $\beta$ factor, in the acceleration term, is related to the velocity profile and is defined by $\frac{1}{h} \int_{0}^{h} u^{2}(y) d y=\beta\left(\frac{1}{h} \int_{0}^{h} u(y) d y\right)^{2}$. It is generally taken to be equal to 1 , which corresponds to the exact value for plug flows [49]. It can be calculated provided an assumption on the shape of the velocity profile: $\beta=4 / 3$ for linear velocity profiles and $\beta=5 / 4$ for Bagnold velocity profiles [51]. The $k$ factor, in the thickness gradient term, is the ratio of the normal stress $\sigma_{x x}$ to the normal stress $\sigma_{y y}$, classically introduced for dense granular flows [49, 50]. For steady dense granular flows, $k$ can be chosen equal to 1 (isotropic fluid-like behavior) 
as shown by previous studies [32]. From Eq. (4), we could estimate the effective friction coefficient at a given position $x_{0} / d$ :

$$
\mu^{*}\left(x_{0}\right) \simeq \tan \theta-\left[\frac{\triangle h}{\triangle x}\right]_{x=x_{0}}-\frac{\beta}{g h \cos \theta}\left[\frac{\triangle\left(h \bar{u}^{2}\right)}{\triangle x}\right]_{x=x_{0}}
$$

We performed our calculations with $\beta=1, \triangle x=200 d, \triangle h=h_{x=x_{0}-100 d}-h_{x=x_{0}+100 d}$ and $\triangle\left(h \bar{u}^{2}\right)=\left(h \bar{u}^{2}\right)_{x=x_{0}-100 d}-\left(h \bar{u}^{2}\right)_{x=x_{0}+100 d}$. Fig. 2 shows the variation of the effective friction coefficient $\mu^{*}$ versus the macroscopic inertial number $I$ for different positions $x / d$. All the data collapse into a single curve whatever the position $x / d$ and the slope, which shows that the existence of a unique relationship between $\mu^{*}$ and $I$ is still valid for accelerating flows investigated here. We also reported the laws from Eq. (2) with $b=0.5$ and from Eq. (3). These laws should be valid for steady and uniform flows when $\tan \theta=\mu^{*}$. This is the case for the Eq. (2) which predicts fairly well the data for $\theta<\theta_{\max }$ (low $I$ values) but fails for larger values of $\theta$ (large $I$ values). The Eq. (3) was shown to be in good qualitative agreement with the data in the sense it predicts the asymptotic saturation of the friction at large values of $I\left(\mu^{*} \rightarrow \mu_{\max }\right)$. We reported the equation prediction for three values of $\theta_{\max }$ : $24^{\circ}, 26^{\circ}$ and $28^{\circ}$. Results show that a low value of $\theta_{\max }$ gives a good prediction at low $I$ but fails in predicting the saturation friction, whereas a higher $\theta_{\text {max }}$ gives a relevant prediction at large $I$ but fails in predicting the data at low $I$. The inset graph in Fig. 2 gives $\mu^{*}$ versus $I$ with $\beta=5 / 4$ for comparison.

Fig. $2 \mathrm{~b}$ shows the mean volume fraction $\bar{\phi}_{3 D}$ versus the inertial number $I$ for different positions $x / d$. Our simulations were performed in two dimensions with spherical beads (no disks). The mean volume fraction $\bar{\phi}_{3 D}$ was calculated from the mean volume fraction $\bar{\phi}_{2 D}$ assuming $\bar{\phi}_{3 D}=\frac{2}{3} \bar{\phi}_{2 D}$ if we compare a sphere of diameter $d$ included in a cube of identical size $d$ to a disk of diameter $d$ included in a square of size $d$. Again, all the data are shown to collapse into a single curve whatever the position $x / d$ and the slope, which supports the existence of a unique relationship between $\phi$ and $I$. Fig. $2 \mathrm{~b}$ presents a comparison of the results to the following law proposed in the literature [29, 48]:

$$
\bar{\phi}=\bar{\phi}_{\max }+\left(\bar{\phi}_{\min }-\bar{\phi}_{\max }\right) I
$$

A good agreement is found for $\theta \leq \theta_{\max }$ (steady and uniform flows) and deviation from this law for $\theta \geq \theta_{\max }$ (slightly non uniform flows), if we consider typical values $\bar{\phi}_{\max }=0.55$ 
and $\bar{\phi}_{\min }=0.4$.

In conclusion, steady and uniform flows $\left(\theta \leq \theta_{\max } \approx 24^{\circ}\right)$ as well as slightly non-uniform flows $\left(\theta \geq \theta_{\max }\right)$ were investigated and characterized with regards to recent new insights on dense granular flows. We also measured the velocity and density profiles over depth and found good agreement with previously published data on dense granular flows down rough inclined planes $[29,32,40]$, i.e., Bagnold-like velocity profiles and a constant volume fraction. We carried out a second set of numerical simulations for which we added a vertical wall normal to the bottom at the location $x / d=900$, as depicted in Fig. 3. The obstacle height $H$ was systematically taken equal to the flow depth $h_{1}$ of the control flow: $H / h_{1}=1$ (for $\theta \geq \theta_{\max }, h_{1}$ was defined at the position $x / d=900$ ). The following analyzes the force on the wall resulting from these dense granular flows.

\section{Mean force on the obstacle}

Fig. 4a shows an example of how the force exerted on the obstacle evolves over a 0.4s time-duration window. The force is characterized by high-frequency fluctuations with possible high amplitudes. We systematically observed a fluctuating chain forces network formed inside the dead zone being the source of the high-frequency fluctuations. Force chains in granular media are highly fluctuating physical processes that have been widely studied in the literature (see, for example, [52-56]). This issue is not discussed in detail in this paper. The total normal force $F_{n}(t)$ on the wall, at a given time $t$, is the sum of each force $f_{n}^{i}(t)$ mobilized at the contact points between the wall and each bead $i$ in contact with the wall:

$$
F_{n}(t)=\sum_{i=1 \ldots n} f_{n}^{i}(t)
$$

where $n$ is the number of beads in contact with the wall at the given time $t$. The number $n$ is closely approximated by the ratio between the obstacle height and the mean particle diameter: $n \sim H / d$. When a bead $j$ in contact with the wall is trapped in a relatively long force chain, the force $f_{n}^{i=j}$ increases substantially and largely contributes to the total force $F_{n}(t)$, which gives a force peak. Such a mechanism can be illustrated in Fig. 4b which gives the change over time of the ratio of the force $f_{n}^{i=i_{\max }}$ to the total normal force $F_{n}(t)$, where $f_{n}^{i=i_{\max }}$ is the instantaneous force which corresponds to the bead exerting the maximum force 
on the wall. When the ratio $f_{n}^{i=i_{\max }} / F_{n}(t)$ is close or equal to one (point $\mathrm{A}$ in Fig. $4 \mathrm{~b}$ ), it means that the total force is mainly due only one bead in contact with the wall and trapped in a force chain. Inversely, when no bead in contact with the wall is trapped in a force chain, each bead has a similar contribution to the total force $F_{n}(t)$ and the ratio $f_{n}^{i=i_{\max }} / F_{n}(t)$ is lower (point B in Fig. 4b).

We represented the moving average over $0.02 \mathrm{~s}$ and $0.1 \mathrm{~s}$ in Fig. 4a. The graph indicates that the moving average tends toward a non-time-dependent hydrodynamic force, one of the main points of the present paper. Fig. 4c shows the mean steady normal force $F_{n}$ on the wall versus the slope inclination $\theta$. The results show that the normal force increases sharply on low slope inclinations (low value of $I$ ) when decreasing the slope inclination. The dramatic increase in force when $\theta$ is close to $\theta_{\min }$ is proof of a transition toward a quasi-static regime and the flow stopping. The dead zone formed upstream of the obstacle tends to move upward indefinitely and the mean force is greatly increased by the weight of the granular material stored upstream of the wall. The mean force is quasi-constant at intermediate slope inclinations. The last data point seems to show a slight increase in force at the highest slope inclination which was investigated (highest value of $I$ ). Here we have a transition toward an rapid - or inertial - regime for which the force is mainly controlled by the flow velocity. These regimes are also visible in the inset in Fig. 4c, which shows the rescaled normal force $F_{n} / F_{0}$ versus the inertial number $I$, where $F_{0}$ is a typical flow force equal to $F_{0}^{d y n}=\frac{1}{\beta} \int_{0}^{h_{1}} \frac{1}{2} \rho_{1} u^{2}(y) d y=\frac{1}{2} \rho_{1} u_{1}^{2} h_{1}$ (dynamic contribution) or

$F_{0}^{h y d r o}=\int_{0}^{h_{1}} \rho_{1} g(h-y) \cos \theta d y=\frac{1}{2} \rho_{1} g h_{1}^{2} \cos \theta$ (hydrostatic contribution). $u_{1}=\int_{0}^{h_{1}} u(y) d y$ is the mean velocity over flow depth $h_{1}$ of the incoming flow of density $\rho_{1}$. The following section proposes a hydrodynamic model to describe this behavior in greater detail and quantify the different contributions to the mean force in each regime defined by the inertial number $I$.

\section{HYDRODYNAMIC MODELING}

\subsection{Momentum conservation in a control volume}

In a steady regime, the variation of momentum in a fixed control volume of fluid $V$ is equal to the sum of the volume forces (the weight of the granular material here) and of the external forces resulting from the elements in contact with the control volume: 


$$
\int_{S} \rho \mathbf{u}(\mathbf{u} \cdot \mathbf{n}) d S=-\int_{S} p \mathbf{n} d S+\int_{S} \sigma \cdot \mathbf{n} d S+\iint_{V} \rho \mathbf{g} d V
$$

We use a single integral symbol for external forces $\left(\int_{S}\right)$ and a double integral symbol for the volume forces $\left(\iint_{V}\right)$ because we consider variables per unit width (in $\mathrm{N} \mathrm{m}^{-1}$ ). $\mathbf{n}$ is the unit vector normal to the surface $S$ of the control volume $V, \rho$ the density, u the velocity, $\mathrm{g}$ the gravity acceleration, $p$ the pressure and $\sigma$ the stress tensor. We apply the momentum conservation on the control volume $V_{0}$ defined on the schematic view given in Fig. 5 . If we consider sections $\left(S_{1}\right)$ and $\left(S_{2}\right)$ defined in Fig. 5, Eq. (8) gives:

$$
\beta Q_{m}^{0}\left(\mathbf{u}_{2}+\mathbf{u}_{1}\right)=\mathbf{P}+\mathbf{P}_{0}+\mathbf{P}_{\mathbf{1}}+\mathbf{P}_{\mathbf{2}}+\mathbf{R}-\mathbf{F}
$$

where $\mathbf{u}_{1}=\frac{1}{h_{1}} \int_{S_{1}} \mathbf{u} d S$ and $\mathbf{u}_{2}=\frac{1}{h_{2}} \int_{S_{2}} \mathbf{u} d S$ are the mean velocities at sections $\left(S_{1}\right)$ and $\left(S_{2}\right)$ defined in Fig. 5, and also shown in Fig. 3. $S_{1}$ is the section normal to the bottom and the incoming flow at the position where the obstacle does not create disturbance in the upstream incoming flow. $S_{2}$ is the section normal to the outgoing flow at the top of the obstacle and making an angle $\alpha$ with the bottom. Mass flow rate conservation gives $Q_{m}^{0}=\rho_{1} u_{1} h_{1}=\rho_{2} u_{2} h_{2}$ where $Q_{m}^{0}$ is the mass flow rate, $h_{1}$ is the thickness of the incoming flow at section $\left(S_{1}\right), \rho_{1}$ its density, and $h_{2}$ is the thickness of the outgoing flow at section $\left(S_{2}\right)$ and $\rho_{2}$ its density. Let us define all the forces involved in momentum conservation. $\mathbf{P}$ is the weight of the control volume: $P_{x}=\rho_{0} g V_{0} \sin \theta$ (component of $\mathbf{P}$ in the $x$-axis direction) and $P_{y}=-\rho_{0} g V_{0} \cos \theta$ (component of $\mathbf{P}$ in the $y$-axis direction). $\rho_{0}$ is the mean density of the granular material in the control volume assumed to be close to the incoming flow density: $\rho_{0} \approx \rho_{1}$. The force $\mathbf{P}_{\mathbf{0}}$ due to the intersitial fluid (air) at the free surface of the flow are ignored: $P_{0} \sim 0$. The pressure force $\mathbf{P}_{\mathbf{1}}$ due to the incoming fluid on section $\left(S_{1}\right)$ is parallel to the bottom: $P_{1 x}=\frac{1}{2} k \rho_{1} g h_{1} \cos \theta\left(P_{1 y}=0\right)$, where $k$ is the earth pressure coefficient classically introduced for dense granular flows $[49,50]$. The pressure force $\mathbf{P}_{\mathbf{2}}$ resulting from the outgoing fluid on section $\left(S_{2}\right)$ is assumed to be negligible $\left(P_{2} \approx 0\right)$. This assumption is argued by the presence of a flying and dilute granular jet (downstream the wall) which does not exert any pressure on the more dense incoming flow at section $\left(S_{2}\right)$ (zero pressure boundary condition). The reaction of the bottom $\mathbf{R}$ has two components. $R_{y}$ is the $y$-axis component and $R_{x}$ is the $x$-axis component corresponding to the mean basal friction force assumed to be proportional to the normal force ( $y$-component of the weight of the volume 
$\left.V_{0}\right): R_{x}=\mu_{z m} P_{y}$. We will discuss this assumption in detail and the meaning of the mean friction $\mu_{z m}$ later in the paper. $\mathbf{F}$ is the mean force exerted on the wall by the granular flow.

Numerical simulations showed a curvature of the free-surface of the flow in the zone of influence of the obstacle. Here, for the sake of simplicity, we assume that the free-surface can be modeled by a straight line between sections $\left(S_{1}\right)$ and $\left(S_{2}\right)$ in Fig. 5 . The disturbance created by the obstacle in the flow is characterized by a free-surface inclined at an angle $\alpha_{s l}$ and the dead zone inclined at an angle $\alpha_{z m}$ with the bottom (see Fig. 5). This assumption and the relation $H=h_{1}$ allow us to estimate the volume $V_{0}$ :

$$
V_{0}=\frac{1}{2} h_{1}\left[\left(2+\frac{\delta_{h}}{\cos \alpha}\right) L-h_{1} \delta_{h}^{2} \tan \alpha\right]
$$

where $L$ is the upstream distance at which the flow is no longer influenced by the obstacle (i.e., the distance between the section $S_{1}$ and the obstacle: see Fig. 5 and also the simulation picture in Fig. 3) and $\delta_{h}=h_{2} / h_{1}$ is the ratio between the flow depths in sections $\left(S_{1}\right)$ and $\left(S_{2}\right)$. The obstacle's influence zone can be determined by the following equation:

$$
L=\frac{h_{1} \delta_{h}}{(\cos \alpha)\left(\tan \alpha_{s l}\right)}
$$

The projection of momentum conservation on the $x$-axis and $y$-axis gives the following equations for $F_{n}$ and $F_{t}$ :

$$
\begin{gathered}
F_{n}=F_{\text {dyn }}+F_{\text {pressure }}+F_{\text {weight-friction }} \\
F_{t}=-\beta \rho_{1} u_{1}^{2} h_{1} \delta_{u} \sin \alpha-\rho_{1} g V_{0} \cos \theta+R_{y}
\end{gathered}
$$

with:

$$
\begin{array}{r}
F_{\text {dyn }}=\beta \rho_{1} u_{1}^{2} h_{1}\left(1-\delta_{u} \cos \alpha\right) \\
F_{\text {pressure }}=\frac{1}{2} k \rho_{1} g h_{1}^{2} \cos \theta \\
F_{\text {weight-friction }}=\rho_{1} g V_{0}\left(\sin \theta-\mu_{z m} \cos \theta\right)
\end{array}
$$

where $\delta_{u}=u_{2} / u_{1}$ represents the variation of mean flow velocity between sections $\left(S_{1}\right)$ and $\left(S_{2}\right)$. As mentioned above, mass flow rate conservation gives $Q_{m}^{0}=\rho_{1} u_{1} h_{1}=\rho_{2} u_{2} h_{2}$. Assuming that the density is unchanged $\left(\rho_{2} \approx \rho_{1}\right)$, we have $\delta_{u} \approx 1 / \delta_{h}$. The following 
proposes simple empirical equations to close the model and determine the angles $\alpha_{s l}, \alpha_{z m}$ and $\alpha$ (defined in Fig. 5), the basal friction $\mu_{z m}$, and the velocity ratio $\delta_{u}$.

\subsection{Free-surface and dead zone angles}

The more inclined the slope is, the higher the free-surface angle upstream of the wall. Therefore, we can assume that $\alpha_{s l}$ is a simple affine function of the slope inclination $\theta$ : $\alpha_{s l}=a \theta+b$, where $a$ and $b$ are empirical coefficients to be determined. Two asymptotic conditions may be considered for each incoming regime: (i) the uniform - dense - regime and (ii) the non-uniform - more dilute - regime. It gives different values of the parameters $a$ and $b$ for each regime. We note $a_{1}$ and $b_{1}$ (respectively, $a_{2}$ and $b_{2}$ ) the coefficients in the dense (respectively dilute) regime. First, let us consider the incoming dense uniform regime for $\theta<\theta_{\max }$. When $\theta=\theta_{\min }$, no steady regime is possible. The influence zone propagates increasingly upstream of the obstacle $(L \rightarrow \infty)$, which corresponds to a value of $\alpha_{s l}$ close to zero. This asymptotic condition gives: $0=a_{1} \theta_{\min }+b_{1}$, which implies $\alpha_{s l}=a_{1}\left(\theta-\theta_{\min }\right)$. When increasing the inclination angle $\theta$, the angle $\alpha_{s l}$ increases until it reaches the critical value $\alpha_{s l}^{c}$ for $\theta=\theta_{\text {max }}$, which gives the asymptotic condition: $\alpha_{s l}^{c}=a_{1}\left(\theta_{\max }-\theta_{\min }\right)$. Second, we consider the incoming non-uniform and dilute regime for $\theta>\theta_{\max }$. We can consider the situation for which the influence zone tends toward zero, which implies that the angle $\alpha_{s l}$ tends toward $\pi / 2$ at a critical slope inclination $\theta_{c}$. This asymptotic condition gives: $\pi / 2=a_{2} \theta_{c}+b_{2}$. $\alpha_{s l}$ may be expressed as: $\alpha_{s l}=a_{2}\left(\theta-\theta_{c}\right)+\pi / 2$. When decreasing the inclination angle $\theta$, the angle $\alpha_{s l}$ decreases until it reaches the value $\alpha_{s l}^{c}$ defined above for $\theta=\theta_{\text {max }}$, which gives: $\alpha_{s l}^{c}=a_{2}\left(\theta_{\max }-\theta_{c}\right)+\pi / 2$. Combining all these asymptotic conditions, we obtain the following changes in $\alpha_{s l}$ according to the slope angle $\theta$ :

$$
\begin{array}{r}
\alpha_{s l}\left(\theta<\theta_{\max }\right)=\frac{\alpha_{s l}^{c}}{\theta_{\max }-\theta_{\min }}\left(\theta-\theta_{\min }\right) \\
\alpha_{s l}\left(\theta>\theta_{\max }\right)=\left(\frac{\alpha_{s l}^{c}-\frac{\pi}{2}}{\theta_{\max }-\theta_{c}}\right)\left(\theta-\theta_{c}\right)+\frac{\pi}{2}
\end{array}
$$

The values of the critical angles $\theta_{c}$ and $\alpha_{s l}^{c}$ will be discussed and determined later in the paper. In section $\left(S_{2}\right)$, the grains at the free surface have a velocity parallel to the free surface and grains at the top of the obstacle (base of the launching downstream jet) have a 
velocity parallel to the line defined by the upstream dead zone. The angle $\alpha$ defined in Fig. 5 can then be approximated by:

$$
\alpha=\frac{\alpha_{s l}+\alpha_{z m}}{2}
$$

Considering that the length of the dead zone formed upstream of the obstacle is identical to the length of the influence zone defined according to the free-surface (length $L$ of the control volume in Fig. 5), the angle $\alpha_{z m}$ defined in Fig. 5 can be determined by the following implicit equation:

$$
\tan \alpha_{z m}=\frac{\tan \alpha_{s l}}{1+\frac{h_{1}}{H}\left(\frac{\delta_{h}}{\cos \left(\frac{\alpha_{s l}+\alpha_{z m}}{2}\right)}+1\right)}
$$

\subsection{Variation in velocity and depth}

Incoming flows encounter a local decreased slope created by the dead zone upstream of the obstacle, which leads to a decrease in velocity. The term $\delta_{u}$ defined in momentum conservation (Eq. (9)) is then smaller than one: $\delta_{u}=u_{2} / u_{1}<1$. We do not have precise measurements of the velocity profiles at section $S_{2}$, which would allow us to test a possible theoretical prediction of $\delta_{u}$ based on the assumption of Bagnold velocity profiles at sections $S_{1}$ and $S_{2}$. Future work is needed in that sense. Here we simply propose an empirical law based on the following statement: the larger the deflecting angle $\alpha$, the higher the expected velocity decrease is. Thus, we simply assume that the relative velocity reduction is proportional to the angle $\alpha: \triangle u / u_{1}=\left(u_{1}-u_{2}\right) / u_{1}=\kappa \alpha$, where $\kappa$ is a coefficient to be determined. This equation allows us to quantify $\delta_{u}$ :

$$
\delta_{u}=1-\kappa \alpha
$$

Let us note that the free-surface angle $\alpha$ is strongly correlated to the obstacle's influence zone $L$ : the larger $\alpha$, the longer $L$. We could have also proposed a simple relation between $\delta_{u}$ and $L$. By mass flow rate conservation and assuming an unchanged density, the flow depth is increased $\left(\delta_{h}=h_{2} / h_{1}>1\right)$ and can be approximated by:

$$
1 / \delta_{h} \approx 1-\kappa \alpha
$$


The $\kappa$ coefficient is a model parameter that will be discussed later.

\subsection{Basal friction in the dead zone}

The quantification of the basal friction $\mu_{z m}$ is a crucial and non-trivial point. Let us note $T_{b}$ the basal friction force. If we consider grains at the base of the flow at section $\left(S_{1}\right)$, these grains are in movement and the basal friction force is known according to Coulomb sliding condition: $T_{b}=(\tan \theta) P_{y}$ for $\theta_{\min }<\theta<\theta_{\max }$ (steady and uniform flows). For

slightly accelerating flows at slopes inclinations larger than $\theta_{\text {max }}$, the basal friction force $T_{b}$ at section $S_{1}$ is slightly smaller than $(\tan \theta) P_{y}$ (positive flow-depth gradient in the $x$-axis direction). If we consider motionless grains at the base of the flow immediately upstream of the obstacle, the basal friction is locally undetermined (the non-sliding condition gives: $\left.T_{b}<\mu_{z m} P_{y}\right)$. The basal friction $\mu_{z m}$ is then expected to vary between a value equal to $\mu^{*}$ ( $\mu^{*}=\tan \theta$ for steady and uniform flows and $\mu^{*}<\tan \theta$ for gradually accelerating flows) at the beginning of the dead zone and a value which is less and less determined when the obstacle is approached. For simplicity reasons, the constant value that can be given will be discussed below.

\section{NUMERICAL DATA COMPARED TO HYDRODYNAMIC MODELING}

\subsection{Calibration}

To compare the predictions of the proposed hydrodynamic model to the data from discrete numerical simulations, we need to validate the empirical laws proposed to close the model and to determine the different parameters introduced in the model.

A first empirical law has been proposed for the prediction of the angle $\alpha_{s l}$. Fig. 6a gives the angle $\alpha_{s l}$ versus the inclination angle $\theta$. Each group is clearly described by a linear fit and allows us to determine the coefficients $a_{i}$ and $b_{i}$ defined previously. Identifying these coefficients to Eqs. (17) and (18), we can obtain the critical angles $\alpha_{s l}^{c}$ and $\theta_{c}$. We have both unknown parameters and four equations, giving the following results: $\alpha_{s l}^{c} \approx \theta_{\min }$ and $\theta_{c} \approx \pi / 2$. Eqs. (17) and (18) can be then expressed: 


$$
\begin{array}{r}
\alpha_{s l}\left(\theta<\theta_{\max }\right)=\frac{\theta_{\min }}{\theta_{\max }-\theta_{\min }}\left(\theta-\theta_{\min }\right) \\
\alpha_{s l}\left(\theta>\theta_{\max }\right)=\frac{\pi}{2}-\left(\frac{\theta_{\min }-\pi / 2}{\theta_{\max }-\pi / 2}\right)(\pi / 2-\theta)
\end{array}
$$

Fig. 6b shows the angle $\left(\theta-\alpha_{z m}\right)$, which corresponds to the mean angle of the dead zone with the horizontal (see Fig. 5), versus the slope inclination $\theta$. $\left(\theta-\alpha_{z m}\right)$ is remarkably close to $\theta_{\text {min }}$ whatever the slope inclination. The angle $\alpha_{z m}$ can be approximated as follows: $\alpha_{z m} \approx \theta-\theta_{\text {min }}$, instead of using the implicit Eq. (20). The prediction of Eqs. (23) and (24) compared to the angle $\alpha_{s l}$ estimated from numerical simulations are also reported in Fig. 6b. The linear law proposed to describe $\alpha_{s l}$ is in quite good agreement with the numerical data. The inset graph in Fig. 6b shows the numerical angles compared to proposed predictions in terms of $\alpha_{z m}$ and $\alpha_{s l}$ as a function of $I$. This plot is obtained by considering the value of the inertial number that the flow would have in absence of obstacle at $x / d=900$, corresponding to the position of the obstacle.

The relation $\theta-\alpha_{z m}=\theta_{\min }$ gives a simple equation (instead of Eq. (11), which demands knowledge of $\delta_{h}$ and $\alpha_{s l}$ ) to estimate the length $L$ of the obstacle's influence zone. Indeed, we can consider that it is identical to the length of the dead zone (as shown in Fig. 3), which gives: $L \approx H / \tan \left(\theta-\theta_{\min }\right)$. Fig. 6c shows the prediction of the latter equation compared to the length of influence measured directly from numerical simulations. Predictions are satisfying regarding the simple assumptions made. $L$ was estimated graphically using the simulation pictures similar to the typical picture depicted in Fig. 3. It is the distance between the obstacle and the section $\left(S_{1}\right)$, and coincides quite well with the length of the dead zone represented by grains with a velocity smaller than a threshold speed $v_{t}$ (see black colored grains in Fig. 3). This threshold velocity was typically chosen equal to $5 \%$ of the depth-averaged velocity of the granular flow in absence of obstacle $\left(v_{t} \approx 0.05 \bar{u}\right)$.

A second empirical law has been proposed to predict the velocity ratio $\delta_{u}$. It implies a $\kappa$ coefficient difficult to determine. $\kappa$ is expected to vary and display different behaviors in the different flow regimes (dilute, dense and quasi-static regimes). One approximation considers the value of $\kappa$ approximated from the dilute regime for which collisional interactions are dominant. If the effects of the ambiant fluid are not considered, the only source of velocity reduction in the dilute regime stems from collisions between grains and is expected to be proportional to the restitution coefficient. Therefore, we can assume that the $u_{2} / u_{1}$ ratio 
scales as $e$ in the dilute regime. Furthermore, assuming that the length of the dead zone tends to vanish in the dilute regime, it corresponds to a limit angle $\alpha$ of $\pi / 2$, giving the following limit condition according to Eq. (21): $\delta_{u}=e=1-\kappa_{e}(\pi / 2)$. The value of $\kappa$ is then equal to $\kappa_{e}=(1-e) /(\pi / 2)=0.32$ with $e=0.5$. Fig. 6 d shows the prediction of Eq. (22) with $\kappa=\kappa_{e}=0.32$ compared to the depth ratio $h_{2} / h_{1}$ measured directly from numerical simulations. The prediction from the simple law proposed for the depth ratio $\delta_{h}$, is not perfect, but the order of magnitude given by this $\kappa_{e}$ value is satisfying regarding the crude assumption made. The assumption of unchanged density probably has an effect on results. Density $\rho_{2}$ at the top of the obstacle (free boundary and granular jet at section $S_{2}$ ) may be smaller than density $\rho_{1}$, which could explain why the flow depth ratio is underestimated.

Another parameter in the model is the basal friction $\mu_{z m}$. We measured shear and normal basal stresses directly from numerical simulations. Fig. 7a shows the ratio between basal shear and normal stresses $\tau_{x y} / \sigma_{y y}$ versus the position $x / d$ upstream of the obstacle (example for $\left.\theta=26^{\circ}\right)$. Outside of obstacle's influence zone $(-x / d>L / d$ with $x<0$ in Fig. 7a), the friction is close to $(\tan \theta)$ as was expected, and it decreases when approaching the obstacle with high fluctuations close to the obstacle. The variation of $\tau_{x y} / \sigma_{y y}$ is nontrivial. We interpolated all the curves by polynomial functions, named $P_{\mu}(x)$, which allowed us to estimate an averaged friction. $\mu_{z m}$ is estimated from the averaged value obtained at a distance $1 / 3 L$ upstream of the obstacle: $\mu_{z m}=P_{\mu}(x=-1 / 3 L)$ with $x<0$ (Fig. 7a). It corresponds to the position of the dead zone's center of gravity assumed to be triangular in shape. Fig. $7 \mathrm{~b}$ gives $\mu_{z m}$ versus $I$. The values measured are relatively constant and found to be very close to the value calculated from the minimum angle $\theta_{\text {min }}$, which is also reported in Fig. 7b. In spite of the complex force chains network inside the dead zone leading to high fluctuations of the local basal friction $\tau_{x y} / \sigma_{y y}$, it is possible to consider a continuum approach and define an averaged basal friction $\mu_{z m}$ equal to $\left(\tan \theta_{\min }\right)$. This value seems compatible with the observed geometry of the dead zone provided that the angle of the dead zone with the horizontal $\left(\theta-\alpha_{z m}\right)$ is equal to $\theta_{m i n}$, as discussed above (see Fig. 6b) and shown again in the inset graph in Fig. 7b.

All the parameters are now determined, which allows us to compare the predictions of the hydrodynamic analytical model to the numerical data from discrete simulations in terms of the force exerted on the wall. 


\subsection{Quantitative comparison}

Fig. 8a shows the normal force on obstacle $F_{n}$ versus the slope inclination $\theta$ obtained from both the hydrodynamic modeling approach and the data from discrete numerical simulations. Fig. $8 \mathrm{~b}$ shows the same results in terms of the rescaled force $F_{n} / F_{0}$ versus the inertial number $I$, where $F_{0}=F_{0}^{d y n}$ or $F_{0}=F_{0}^{\text {hydro }}$. The analytical prediction is found to be in very good agreement with the numerical data provided the parameters discussed above: $\beta=5 / 4$ (Bagnold-like velocity profile), $\kappa_{e}=(1-e) /(\pi / 2)$ with $e=0.5$ and $\theta_{\min }=14^{\circ}$. We proposed a constant value for $\kappa$ derived from the dilute regime where collisions are dominant with a velocity reduction scaling such as the restitution coefficient $e$. Even if $\kappa$ depends on $e$ and $\mu$ also in the dense granular regime, the results are satisfactory to describe the mean force in this regime because $\delta_{u}$ and $\delta_{h}$ (depending on $\kappa$ ) have only a slight effect on the force estimation in this regime. The value of $k$, representing the ratio of the normal stress $\sigma_{x x}$ to the normal stress $\sigma_{y y}$, was chosen equal to 1 , which corresponds to isotropic material conditions. A value derived from a Mohr-Coulomb plasticity [49, 57] does not provide a better prediction, similar to the results from previous studies [21, 32].

Fig. 8a also reports the different contributions to the normal forces given by Eq. (12). At high inertial numbers $I$, the contribution from the momentum term $\left(F_{d y n}\right)$ is dominant, proving an inertial regime. At low inertial numbers $I$, the pressure term $\left(F_{\text {pressure }}\right)$ and the difference between weight and basal friction $\left(F_{\text {weight-friction }}\right)$ become dominant contributions. In the dense regime $(0<I<0.3-0.4)$, Fig. $8 \mathrm{~b}$ shows that the force scales like the

hydrostatic force $F_{0}^{\text {hydro }}$ but it is four times greater than $F_{0}^{\text {hydro }}$. The contribution of the term $\left(F_{\text {weight-friction }}\right)$ to the total normal force $F_{n}$ is dominant because of the large increase in length of the dead zone, as shown in Fig. 6c.

An important result from discrete simulations, shown by the Fig. 4a, is that the tangential force $F_{t}$ on the obstacle is found to be close to zero whatever the slope inclination: $F_{t} \approx 0$. From Eq. (13), we can estimate the reaction of the bottom in the $y$-axis direction $\left(R_{y}\right)$. Fig. $8 \mathrm{c}$ shows the reaction of the bottom $R_{y}$ versus the inertial number $I$. The reaction $R_{y}$ is strongly increased at low inertial numbers because $-P_{y}=\rho_{1} g V_{0} \cos \theta$ is very large (the influence zone upstream of the obstacle being very long). This behavior is proof of the transition toward the quasi-static regime and prevents the occurrence of a steady flow regime when approaching the angle $\theta_{\min }$. In this quasi-static regime, the momentum force 
in the $y$-axis direction is negligible and the reaction $R_{y}$ is equal to the weight of the material upstream of the obstacle in the $y$-axis direction: $\beta \rho_{1} u_{1}^{2} h_{1} \delta_{u} \sin \alpha<<R_{y}=-P_{y}$. At higher inertial numbers, there exists a critical value $I_{c}$ for which the reaction of the bottom $R_{y}$ is equal to zero (see inset in Fig. 8c), which indicates that the momentum force in the $y$-axis direction is exactly balanced by the weight of the material stored upstream of the obstacle in the $y$-axis direction. Above $I_{c}$, the three contributions are balanced according to the Eq. (13) and $R_{y}<0$. The fact that $F_{t}$ is found to negligible, and the consequences it has on the prediction of the bottom reaction $R_{y}$, should be further studied by initiating experimental studies.

\section{CONCLUSION}

This paper has described the results on the mean force on a vertical wall caused by freesurface dense granular flows down an inclined plane. We presented numerical data from 2D spherical particle discrete simulations performed for a wide range of slopes. The normal force on the obstacle was characterized by high-frequency fluctuations with high amplitudes due to a fluctuating force chains network inside the dead zone formed upstream of the wall. This paper has focused on the mean normal force. We considered a hydrodynamic modeling approach based on momentum conservation in a control volume. The analytical prediction was compared to numerical data and quite accurately reproduced the behavior of the force. Although the numerical simulations display a more complex shape of the dead zone and the free-surface upstream of the obstacle (particularly when approaching the quasi-static regime), we assumed a triangular shape for the dead zone. These simple assumptions, made to close the theoretical model, provided good results. Indeed, the mean angle of the dead zone and the length of the influence zone upstream of the obstacle were captured quite well by the simple laws proposed. The predictions of the angle $\alpha_{s l}$ and of the variation in depth were not perfect and will need further investigation. However, we believe that the hydrodynamic analytical model proposed in this paper with simple empirical arguments to close the model is strikingly satisfying in the sense that it is able to predict the exact order of magnitude of the mean granular force computed from discrete numerical simulations if relevant values are provided for the parameters needed in the model. All these parameters were estimated from simple assumptions with no fitting processes. We can conclude that the dynamics 
of the incoming flow $\left(u_{1}, h_{1}\right.$ and $\left.\phi_{1}\right)$ and the granular material properties $\left(\theta_{\text {min }}=14^{\circ}\right.$, $k=1, \beta=5 / 4$ and $e=0.5$ here) made it possible to estimate the mean steady granular normal force on a vertical obstacle for uniform incoming flows $\left(\theta_{\min }<\theta<\theta_{\max }\right)$ but also for gradually accelerated flows $\left(\theta>\theta_{\max }\right)$. Besides numerical simulations showed that the mean tangential force exerted on the wall is close to zero, which could initiate future experimental studies. Beyond this analytical model, this study provides a new example of the ability of hydrodynamic modeling approaches to describe the mean macroscopic behavior (kinematics and depth-averaged force) of an assembly of rigid grains. It has to be kept in mind that the continuum model proposed in this paper has been developed on the basis of stationary flow conditions (recirculating flows) with an obstacle height close to the incoming flow depth. Neither the force due to rapid granular flows impinging high walls in the presence of a upward traveling shock wave [17], nor the peak impact force due to a granular avalanche front, can be reproduced by the continuum model proposed in this paper. Further investigations will be devoted to the situation of dense granular avalanches (finite volume) overflowing small planar obstacles, in order to see if the continuum model is able to catch the force exerted by the decelerating avalanche tail (after the occurrence of the peak impact force).

\section{Acknowledgments}

The authors acknowledge financial support from the French National Research Agency (ANR-MONHA). The authors are grateful to Mohamed Naaim for fruitful discussions and to David Bertrand for his experience and help in conducting the numerical simulations with the commercial code PFC.

[1] U. Tuzun, and R.M. Nedderman, Chem. Eng. Sci. 40, 337 (1985).

[2] R.M. Iverson, Reviews of Geophysics 35 (3), 245 (1997).

[3] B. Sovilla, M. Schaer, M. Kern, and P. Bartelt, J. Geophys. Res. 113, F01010 (2008).

[4] T. Faug, P. Gauer, K. Lied, M. Naaim, J. Geophys. Res. 113, F03009 (2008).

[5] K. Wieghardt, Mech. Res. Commun. 1, 3 (1974).

[6] K. Wieghardt, Ann. Rev. Fluid. Mech. 7, 89 (1975).

[7] D. Chehata, R. Zenit, and C.R. Wassgren, Phys. Fluids 15 (6), 1622-1631 (2003). 
[8] C.R. Wassgren, J.A. Cordova, R. Zenit, and A. Karion, Phys. Fluids 15 (11), 3318-3330 (2003).

[9] R. Bharadwaj, C. Wassgren, and R. Zenit, Phys. fluids 18 (4), 043301 (2006).

[10] A. Levy, and M. Sayed, Phys. Fluids 19 (2), 023302 (2007).

[11] A. Levy, and M. Sayed, Powder Tech. 181 (2), 137-148 (2008).

[12] V. Buchholtz, and T. Poschel, Granul. Matter 1 (1998).

[13] E.C. Rericha, C. Bizon, M.D. Shattuck, and H.L. Swinney, Phys. Rev. Lett. 88, 014302 (2002).

[14] C.E. Brennen, K. Sieck, and J. Paslaski, Powder Tech. 35, 31 (1983).

[15] S.B. Savage, J. Fluid Mech. 92, 53 (1979).

[16] S. P. Pudasaini, K. Hutter, S. S. Hsiau, S. C. Tai, Y. Wang, and R. Katzenbach, Phys. Fluids 19, $053302(2007)$.

[17] S. P. Pudasaini and C. Kroner, Phys. Rev. E 78, 041308 (2008).

[18] Y.C. Tai, J.M.N.T. Gray, K. Hutter, and S. Noelle, Annal. Glac. 32, 281 (2001).

[19] J.M.N. Gray, Y.-C. Tai, and S. Noelle, J. Fluid Mech. 491, 161 (2003).

[20] M.-C. Chiou, Y. Wang, and K. Hutter, Acta Mech. 175 (14), 105 (2005).

[21] K.M. Hakonardottir and A.J. Hogg., Phys. Fluids 17, 077101 (2005).

[22] J.F. Boudet, Y. Amarouchene, B. Bonnier and H. Kellay, J. Fluid Mech. 572, 413 (2007).

[23] J.M.N.T. Gray, and X. Cui, J. Fluid Mech. 579, 113 (2007).

[24] X. Cui, J.M.N.T. Gray, and T. Johannesson, J. Geophys. Res. 112, F04012 (2007).

[25] R. Albert, M.A. Pfeifer, A.-L. Barabasi, and P. Schiffer, Phys. Rev. Lett. 82, 205 (1999).

[26] I. Albert, P. Tegzes, B. Kahng, R. Albert, J.G. Sample, M. Pfeifer, A.-L. Barabasi, T. Vicsek, and P. Schiffer, Phys. Rev. Lett. 84, 5122 (2000).

[27] I. Albert, J.G. Sample, A.J. Morss, S. Rajagopalan, A.-L. Barabasi, P. Schiffer, Phys. Rev. E 64, $061303(2001)$.

[28] G. Hill, S. Yeung, and S.A. Koehler, Europhys. Lett. 72 (1), 137 (2005).

[29] Y. Forterre and O. Pouliquen, Ann. Rev. Fluid. Mech. 40, (2008).

[30] C. Nouguier, C. Bohatier, J.J. Moreau, F. Radjai, Granul. Matter 2, 171 (2000).

[31] P.A. Cundall, and O.D.L. Strack, Géotechnique 29 (1), 47 (1979).

[32] L. E. Silbert, D. Ertas, G. S. Grest, T. C. Halsey, D. Levine, and S. J. Plimpton, Phys. Rev. E 64, 051302 (2001).

[33] D. Ertas, G.S. Grest, T.C. Halsey, D. Levine, and L.E. Silbert, Europhys. Lett. 56 (2), 214 
(2001).

[34] F. da Cruz F, S. Emam, M. Prochnow, J.N. Roux, and F. Chevoir, Phys. Rev. E 72, 021309 (2005).

[35] J.M. Ting and B.T. Corkum, J. Comp. Civ. Engin. 6, 129 (1992).

[36] C. S. Campbell, J. Fluid Mech. 465, 261 (2002).

[37] H. Hertz, Journal fur die reine und angewandte Mathematik 92, 156 (1881).

[38] G. Lois, A. Lemaitre1, and J.M. Carlson, Phys. Rev. E 72, 051303 (2005).

[39] O. Baran, D. Ertas, T. C. Halsey, G.S. Grest and J.B. Lechman, Phys. Rev. E 74, 051302 (2006).

[40] GDRMidi, E. Phys. J.E 14, (2004).

[41] Itasca consulting (http://www.itascacg.com/pfc/index.php.), Particle Flow Code in 2 Dimensions $\left(\mathrm{PFC}^{2 D}\right.$ version 3.0).

[42] O. Pouliquen, Phys. Fluids 11 (3), 542 (1999).

[43] M. Prochnow, Ph.D. Thesis, Ecole Nationale des Ponts et Chaussées, Marne la vallée, France, 2002.

[44] S.B. Savage, Adv. Appl. Mech. 24, 289 (1984).

[45] C. Ancey, P. Coussot, and P. Evesque, J. Rheol. 43, 1673 (1999).

[46] C. Ancey, Phys. Rev. E 65, 11304 (2002).

[47] P. Jop, Y. Forterre Y, and O. Pouliquen, Nature 441, 72730 (2006).

[48] O. Pouliquen, C. Cassar, P. Jop, Y. Forterre, and M. Nicolas, J. Stat. Mech. P07020 (2006).

[49] S.B. Savage and K. Hutter, J. Fluid Mech. 199, 177-215 (1989).

[50] O. Pouliquen and Y. Forterre, J. Fluid Mech. 453, 133-151 (2002).

[51] Y. Forterre and O. Pouliquen, J. Fluid Mech. 486, 21-50 (2003).

[52] C. Liu et al., Science 269, 513 (1995).

[53] B. Miller, C.O Hern, and R.P. Behringer, Phys. Rev. Lett. 77, 3110 (1996).

[54] S.N. Coppersmith, C.-H. Liu, S. Majumdar, O. Narayan, and T.A. Witten. Phys. Rev. E 53, 4673 (1996).

[55] D.M. Mueth, H.M. Jaeger, and S.R. Nagel, Phys. Rev. E 57, 3164 (1998).

[56] G. Lois, A. Lemaitre, and J.M. Carlson, Phys. Rev. E 76, 021302 (2007).

[57] R. Greeve, T. Koch, and K. Hutter, Proc. R. Soc. London, Ser. A 445, 399 (1994). 
FIG. 1: (a) Thickness $h_{\text {stop }}$ normalized by $d$ versus the slope inclination $\theta$ : open squares represent our numerical data, plain squares represent data from [43] reported also in [40], the continuous line gives the fitted function $h_{\text {stop }}(\theta) / d=B\left[\left(\theta_{\max }-\theta\right) /\left(\theta-\theta_{\min }\right)\right]$ with $\theta_{\min }=24^{\circ}, \theta_{\min }=14^{\circ}$ and $B=2.3$. Inset: sketch of the simulated recirculation system. (b) Velocity profiles measured at $x / d=900$ for control flows at different slope inclinations; the dotted lines give the Bagnold profiles. (c) Inertial number $I$ versus normalized distance $x / d$ for different slope inclinations.

FIG. 2: (a) coefficient of effective friction $\mu^{*}$ versus the inertial number $I$ : our numerical data (with $\beta=1$ ) for different $x / d$ are compared to the predictions from Eq. (2) with $b=0.5$ and Eq. (3) with $I_{0}=0.3$ considering three values of $\theta_{\max }: 24^{\circ}, 26^{\circ}$ and $28^{\circ}$; inset graph: numerical data with $\beta=1$ and $\beta=5 / 4$. (b) Depth-averaged volume fraction $\bar{\phi}$ versus the inertial number $I$ : our numerical data are compared to the linear law from Eq. (6) with $\bar{\phi}_{\max }=0.55$ and $\bar{\phi}_{\min }=0.4$.

FIG. 3: Typical picture of a numerical simulation showing the dead zone formed upstream of the wall overflowed by a granular flow (example for $\theta=24^{\circ}$ ) at a given time (instantaneous picture). We distinguished grains of individual velocity $v$ smaller (black grains) and greater (white grains) than a threshold velocity $v_{t}$, which gives the shape of the so-called "dead zone". The threshold velocity was typically taken equal to $5 \%$ of the depth-averaged velocity of the granular flow in the absence of obstacle: $v_{t} \approx 0.05 \bar{u}$. 
FIG. 4: (a) Change over time for the instantaneous normal (dark gray) and tangential (light gray) components of the force per unit width exerted on the obstacle: example for $\theta=28^{\circ}$. Lines (continuous line for $F_{n}$ and dotted line for $F_{t}$ ) represent the time-averaged values. (b) Change over time of the ratio of the instantaneous force $f_{n}^{i=i_{\max }}$ to the instantaneous normal force $F_{n}(t)$, where $f_{n}^{i=i_{\max }}$ is due to the bead exerting the maximum force among all the beads in contact with the wall. (c) Time-averaged normal force $F_{n}$ per unit of width versus the slope inclination $\theta$. Inset: rescaled normal force $F_{n} / F_{0}$ versus $I$, with $F_{0}=F_{0}^{d y n}=\frac{1}{2} \rho_{1} u_{1}^{2} h_{1}$ and $F_{0}=F_{0}^{\text {hydro }}=\frac{1}{2} \rho_{1} g h_{1}^{2} \cos \theta$. The vertical dashed gray line represents the transition at $\theta=\theta_{\max }$.

FIG. 5: Sketch of the control volume $V_{0}$ (hatched zone) inside which the momentum conservation is applied. $h_{1}$ and $\mathbf{u}_{1}$ are flow depth and mean velocity of the incoming flow at section $\left(S_{1}\right)$. The section $\left(S_{1}\right)$ is normal to the bottom and represents the beginning of the influence's zone of the obstacle. The wall height is $H$ and $L$ is the length of the obstacle's influence zone, i.e., the distance between the section $\left(S_{1}\right)$ and the foot of the obstacle. $h_{2}$ and $\mathbf{u}_{2}$ are flow depth and mean velocity of the flow at section $\left(S_{2}\right)$. The section $\left(S_{2}\right)$ is normal to the main direction of the outgoing flow of velocity $\mathbf{u}_{2}$. We defined the angle $\alpha$ between $\mathbf{u}_{2}$ and the bottom. $\theta$ is the bottom slope. Assuming a triangular shape for the dead zone, we also defined $\alpha_{z m}$ as the angle of the dead zone with the bottom and $\alpha_{s l}$ as the angle of the free-surface (inside $V_{0}$ ) with the bottom.

FIG. 6: (a) Free-surface angle $\alpha_{s l}$ versus the slope inclination $\theta$ : numerical data and linear fits for both dense and dilute regimes. (b), (c) and (d): predictions from the empirical laws compared to numerical data. (b) Angles $\theta-\alpha_{z m}$ and $\theta-\alpha_{s l}$ versus the slope inclination $\theta$; inset graph: angles $\alpha_{z m}$ and $\alpha_{s l}$ versus the inertial number $I$; (c) length of the influence zone upstream of the obstacle normalized by the obstacle height $L / H$ versus $I$; (d) depth ratio $\delta_{h}=h_{2} / h_{1}$ versus $I$ (with $\kappa=\kappa_{e}=0.32$ ).

FIG. 7: (a) Time-averaged ratio between basal shear and normal stresses $\tau_{x y} / \sigma_{y y}$ versus the position $x / d$ (the position $x / d$ is negative): example for $\theta=26^{\circ}$; the dotted line gives the value of $\tan \theta$ and the dashed line $\tan \theta_{\min }$. (b) Estimated values of $\mu_{z m}$ versus $I$ compared to the value $\tan \left(\theta_{\min }\right)$; inset graph: estimated values of $\theta-\alpha_{z m}$ versus $I$ compared to $\theta_{\min }$. 
FIG. 8: (a) Normal force per unit width $F_{n}$ versus the slope $\theta$ (with $\beta=5 / 4$ and $k=1$ ): analytical predictions compared to the numerical data. We also reported the following contributions: $F_{\text {weight-friction }}, F_{\text {pressure }}$ and $F_{\text {dyn }}$. (b) Rescaled force $F_{n} / F_{0}$ versus I from numerical simulations and from the hydrodynamic model. (c) Reaction per unit width of the bottom $R_{y}$, from Eq. (13), versus the inertial number $I$. The $y$-axis component of the weight of the material $\left(P_{y}\right)$ and the $y$ axis component of the momentum force $\left(-\beta \rho_{1} u_{1}^{2} h_{1} \delta_{u} \sin \alpha\right)$ are also reported with $\beta=5 / 4$. Inset: is a zoom on high $I$. 


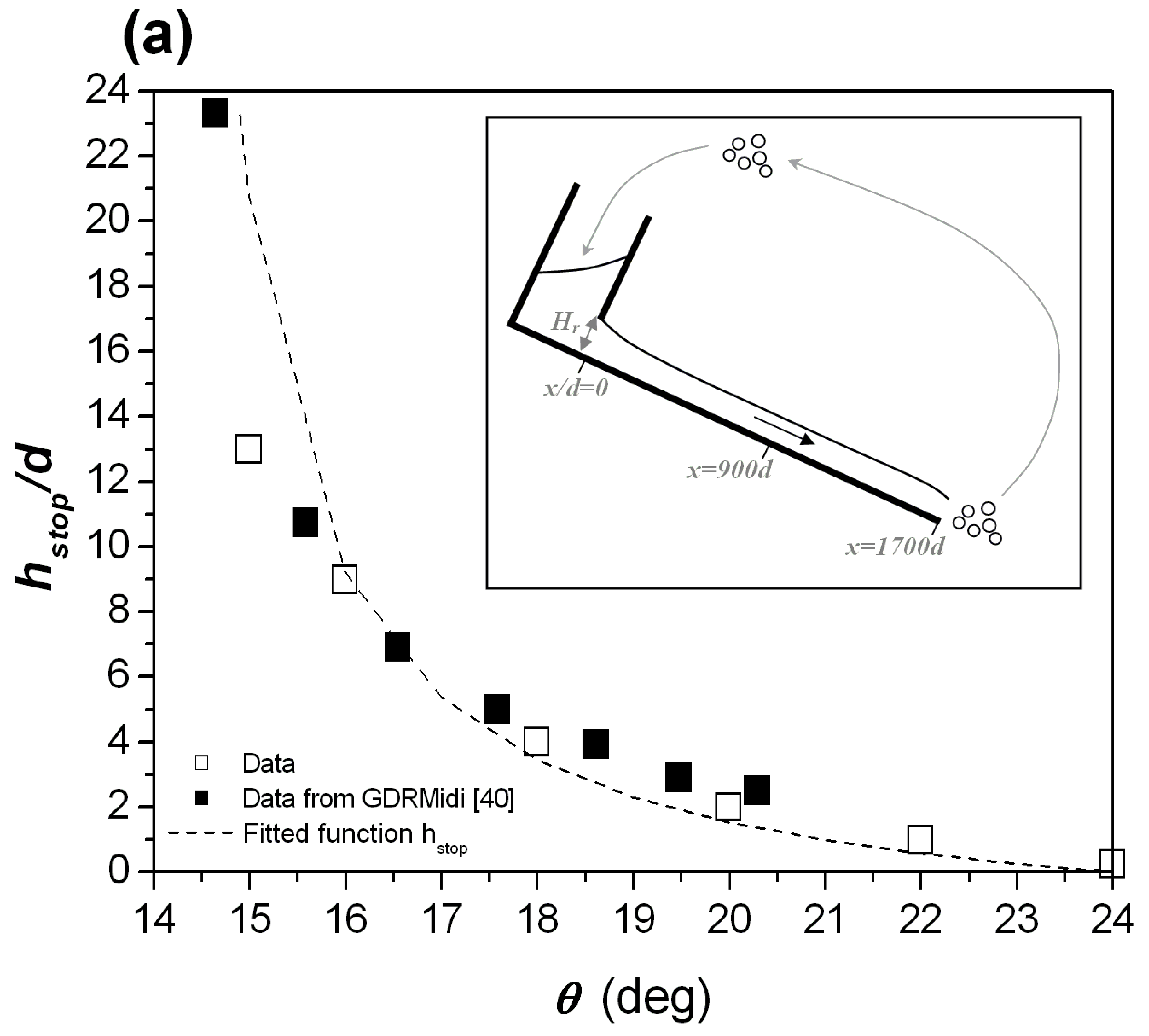




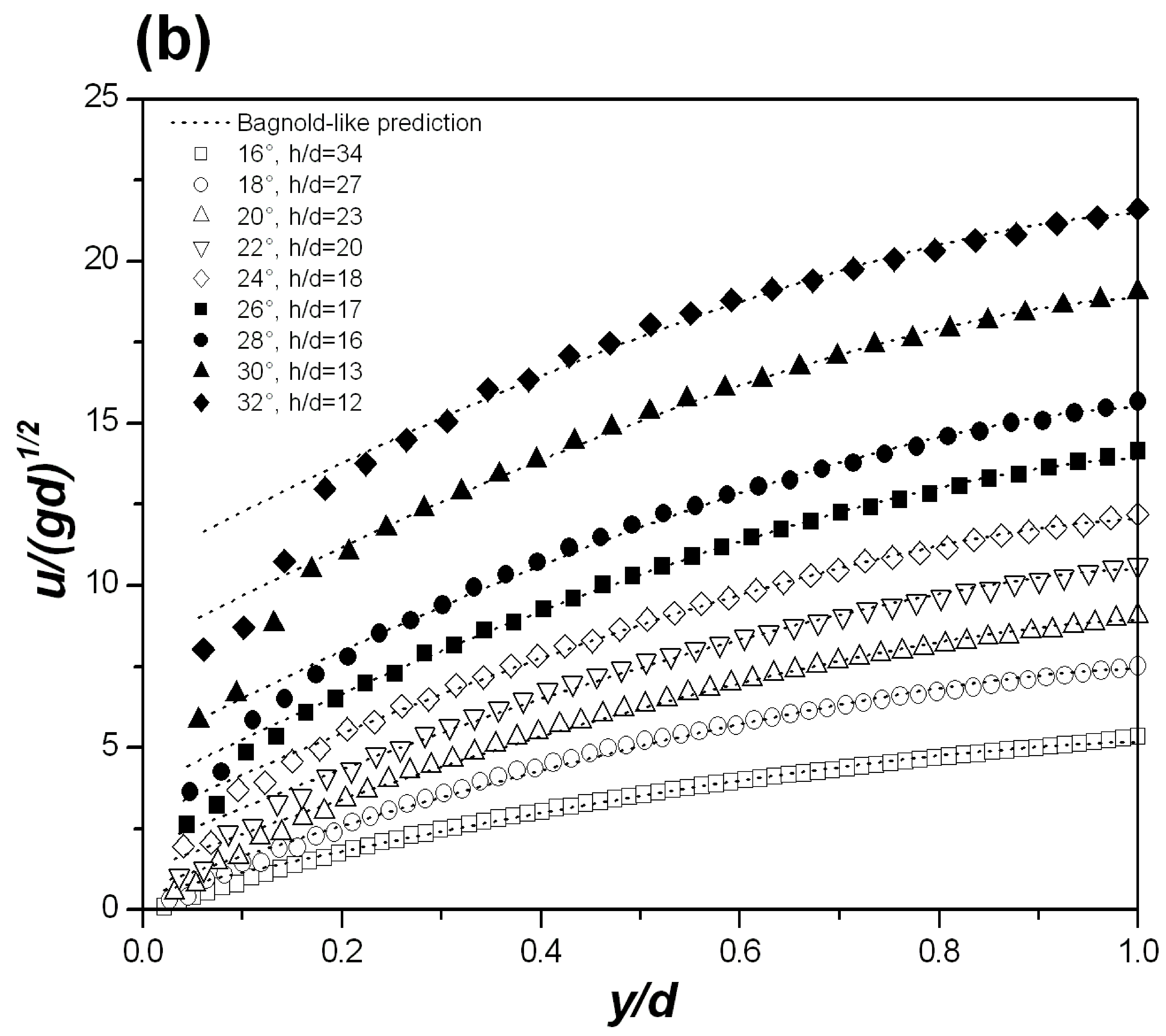




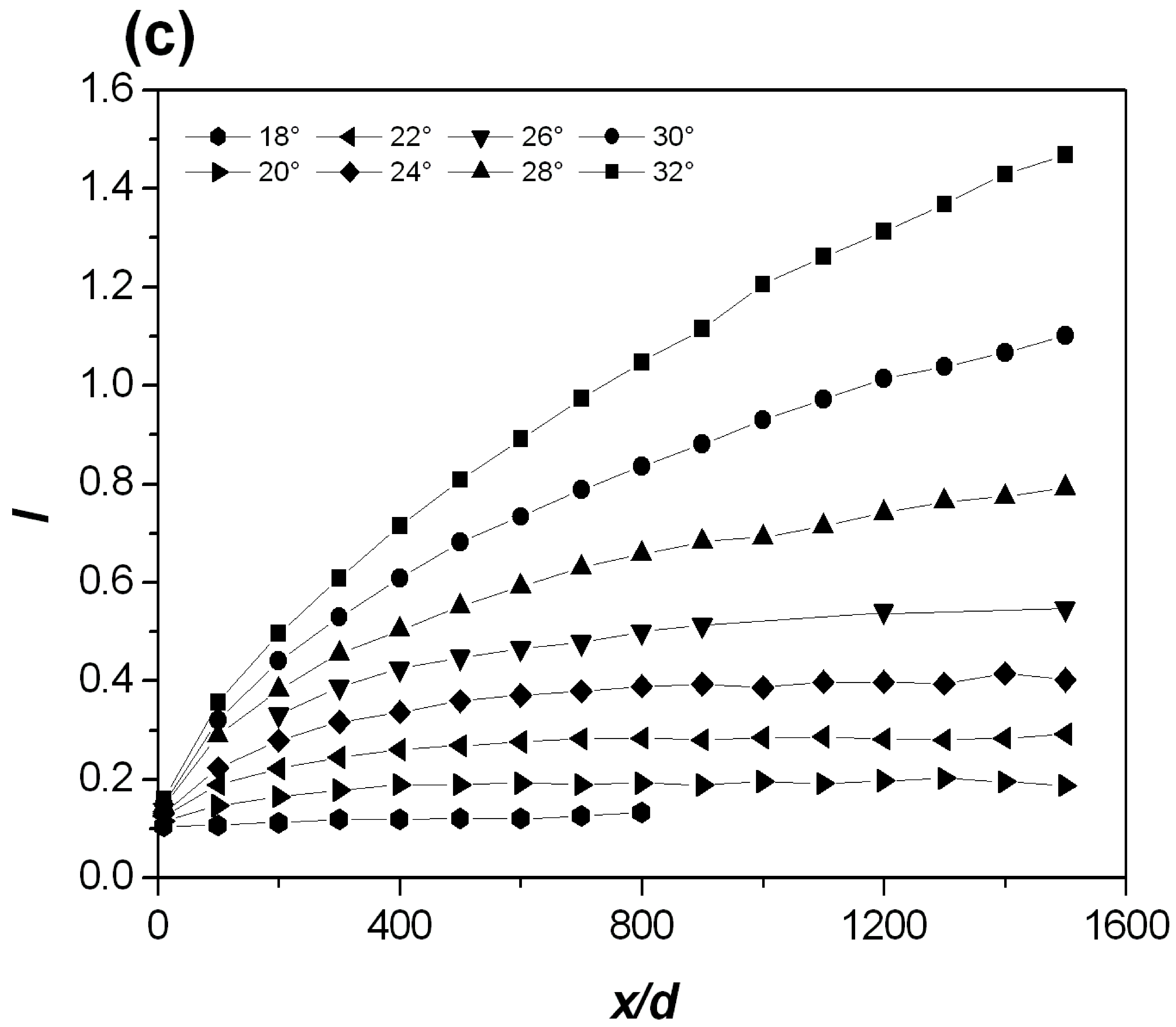




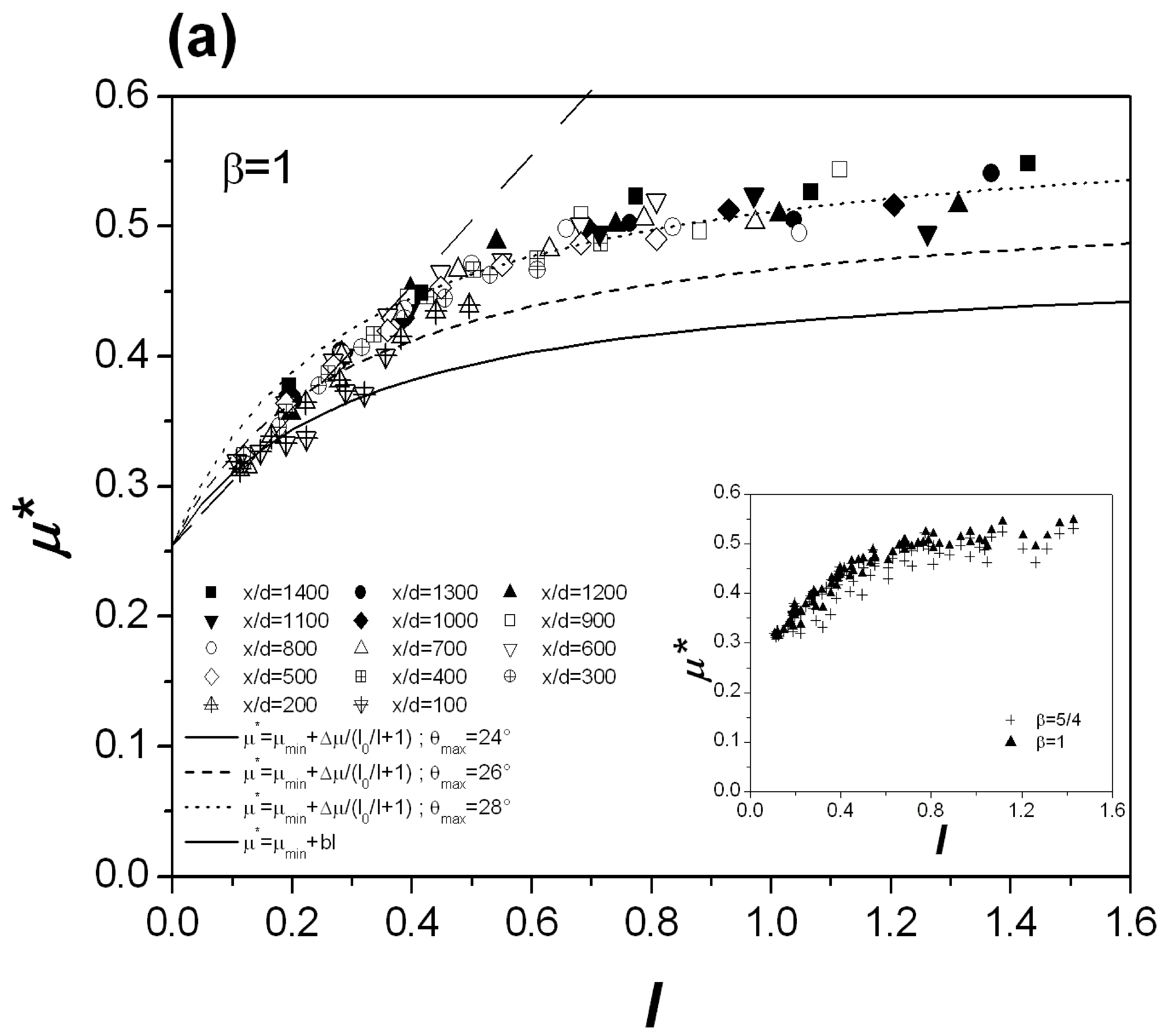


(b)

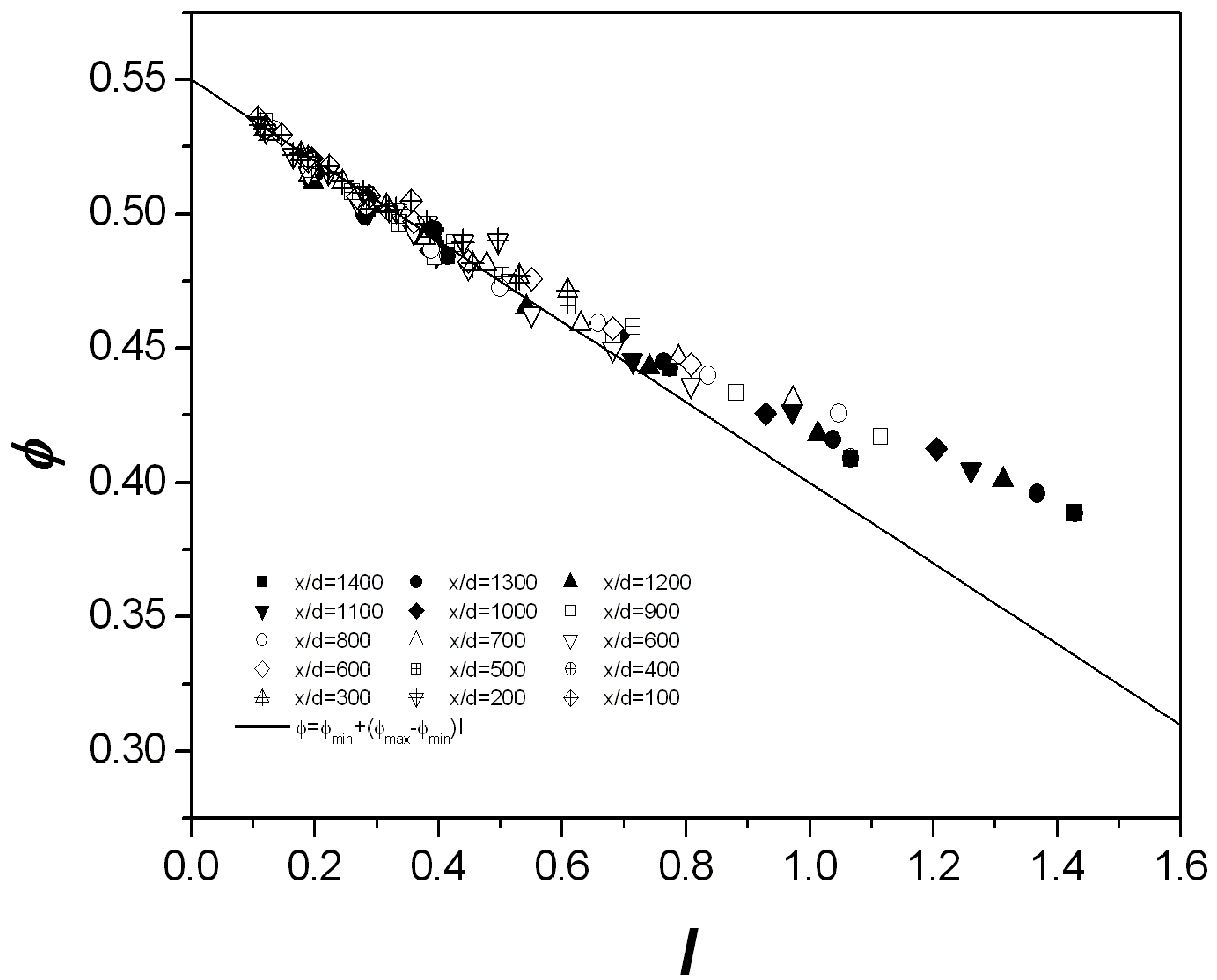




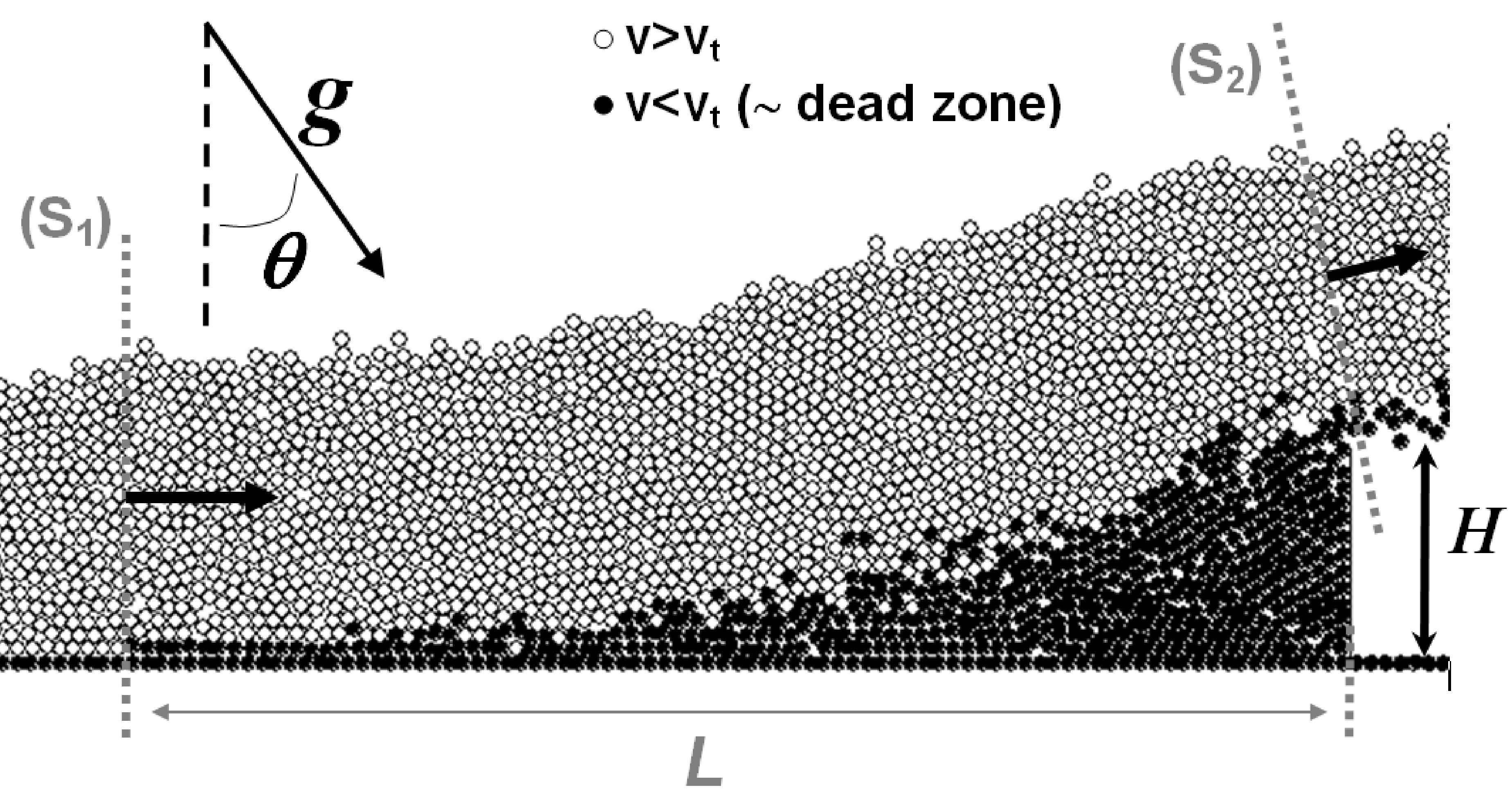


(a)

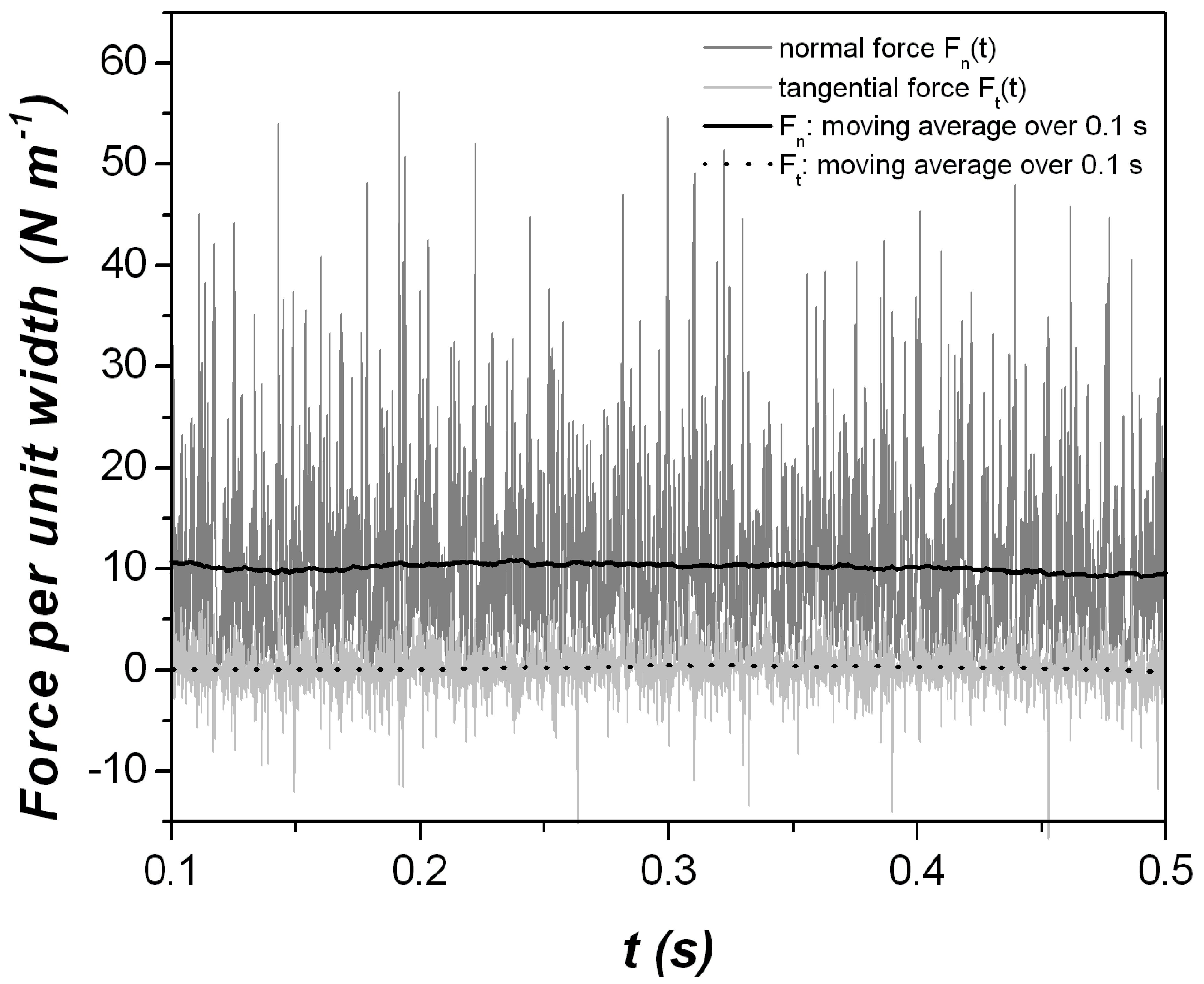




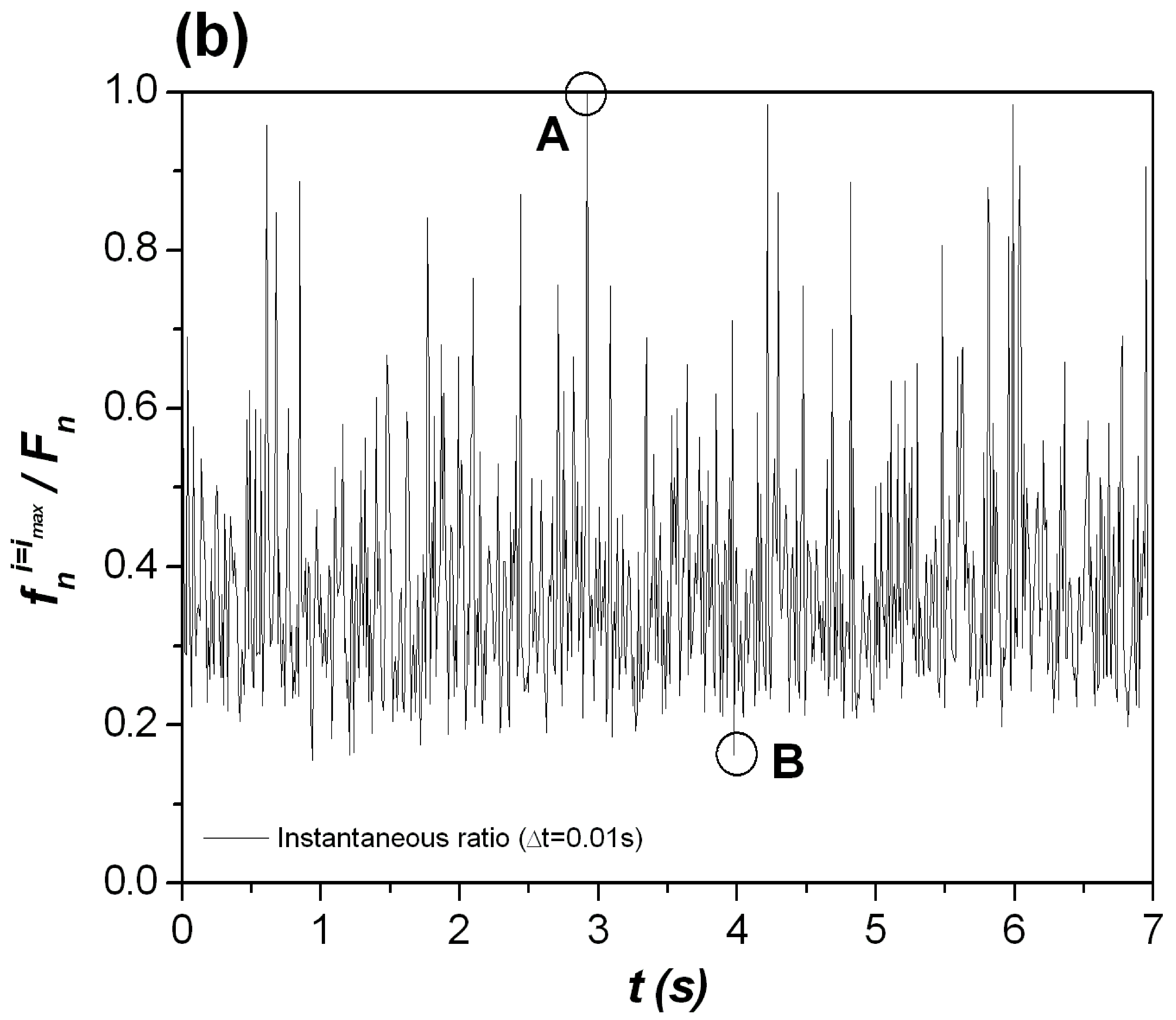




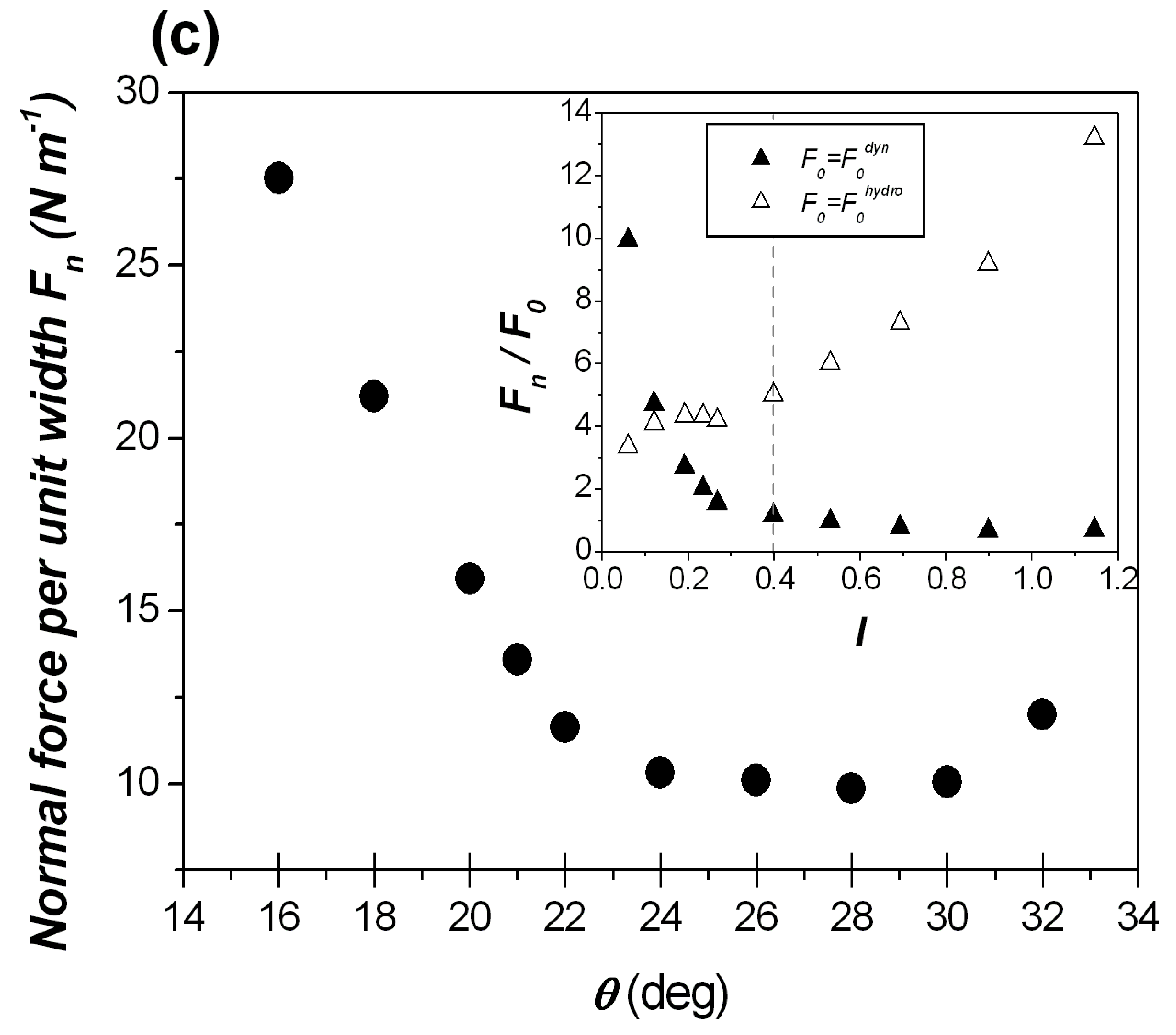




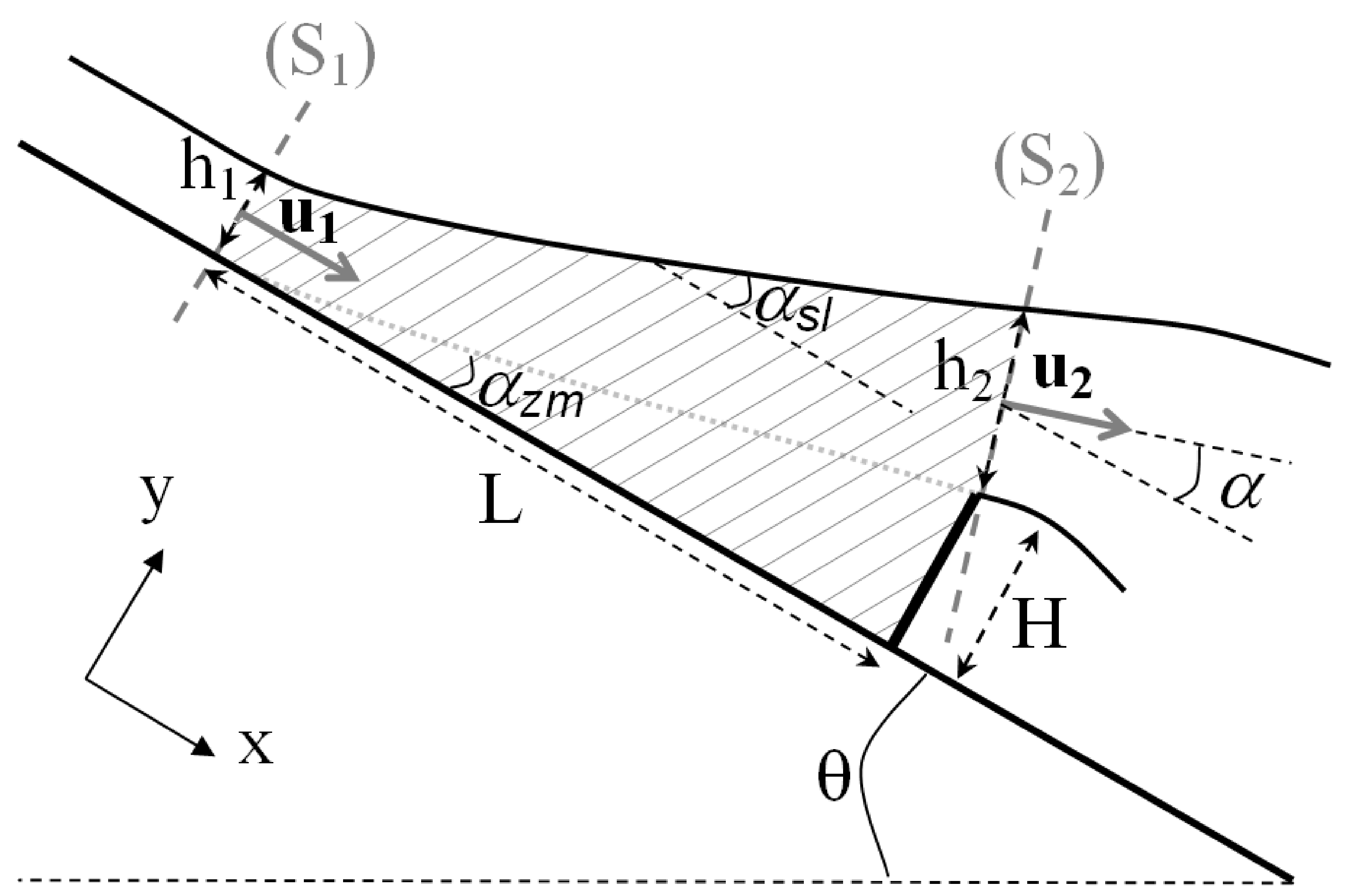




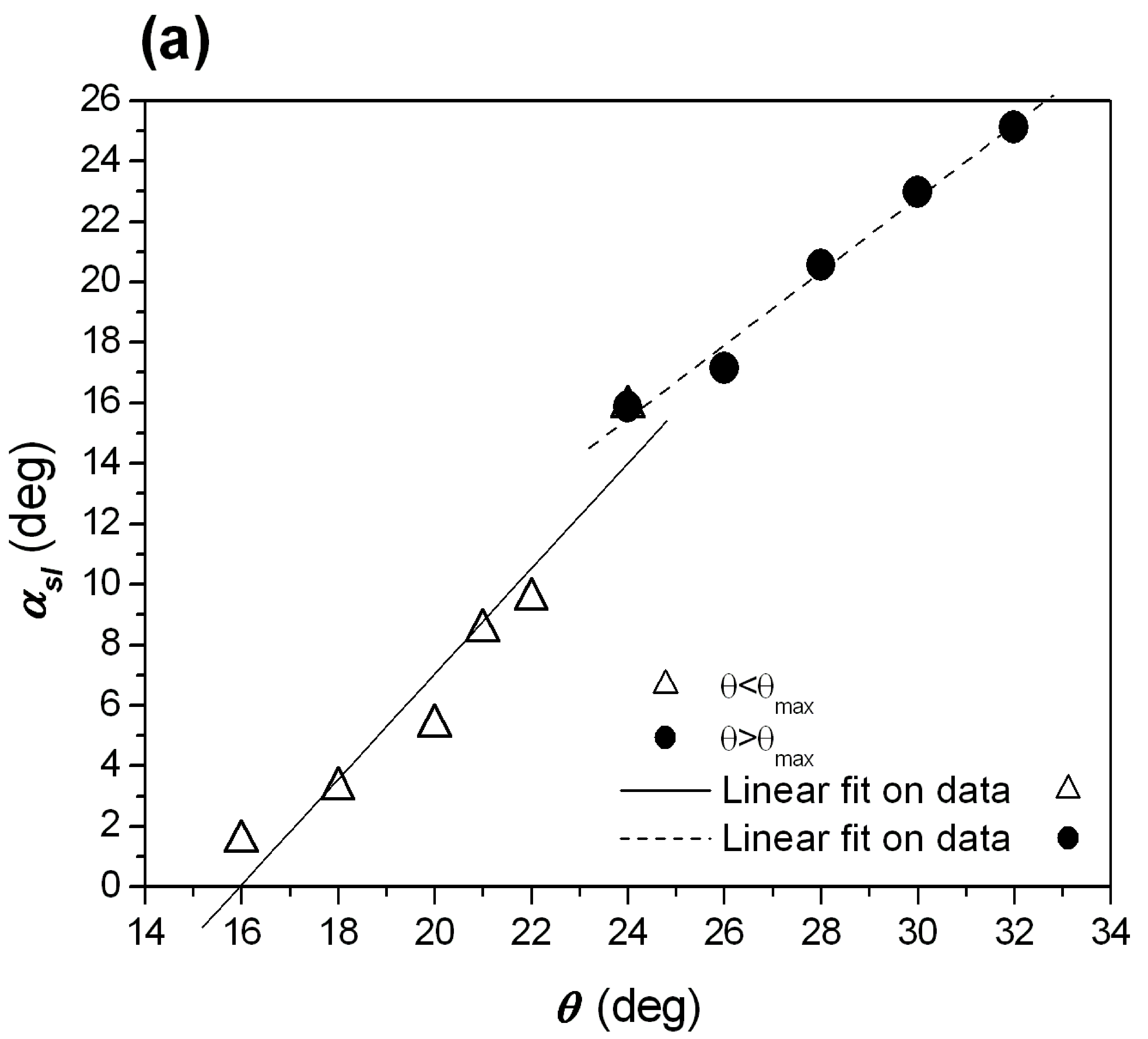




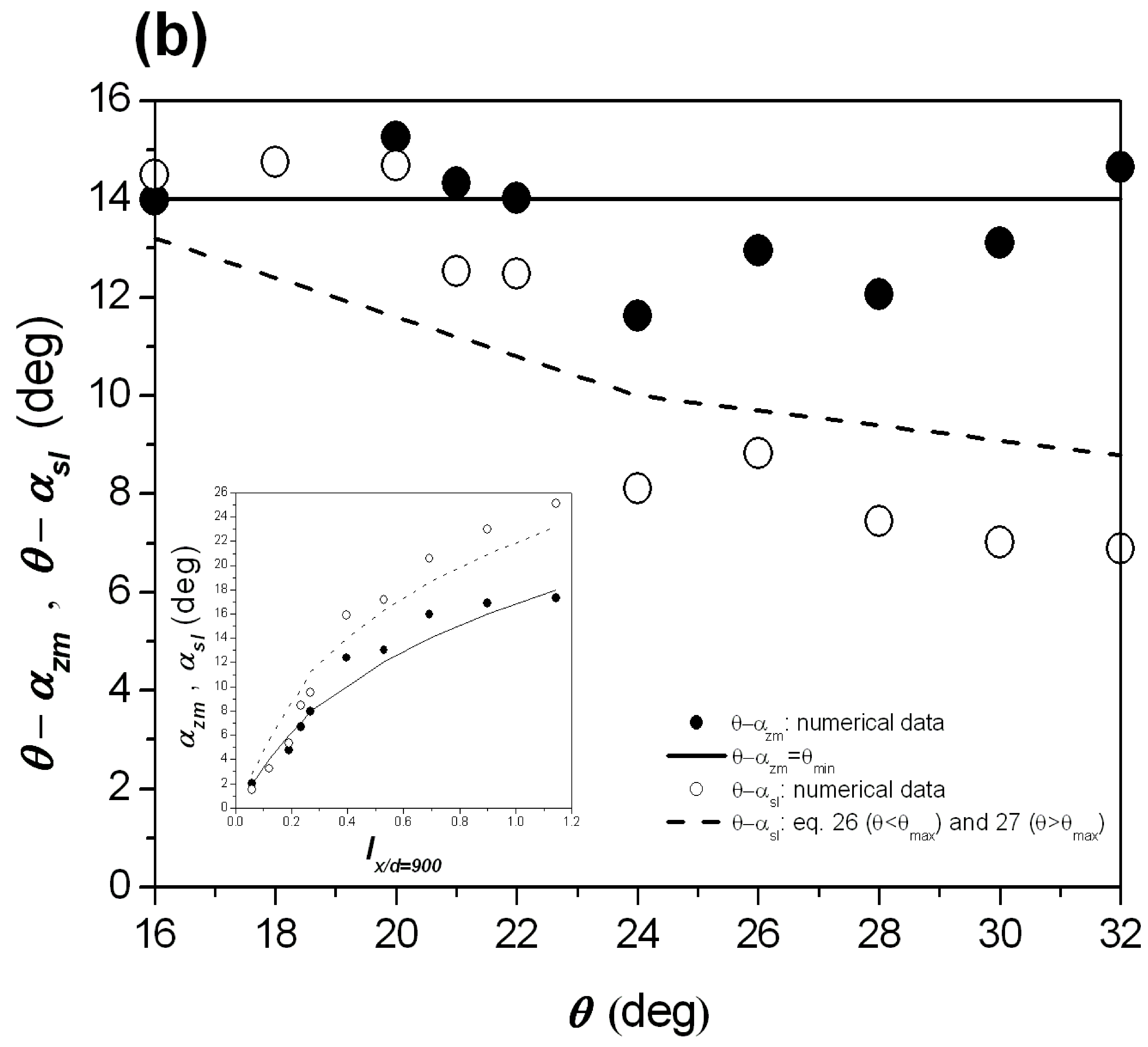


(c)

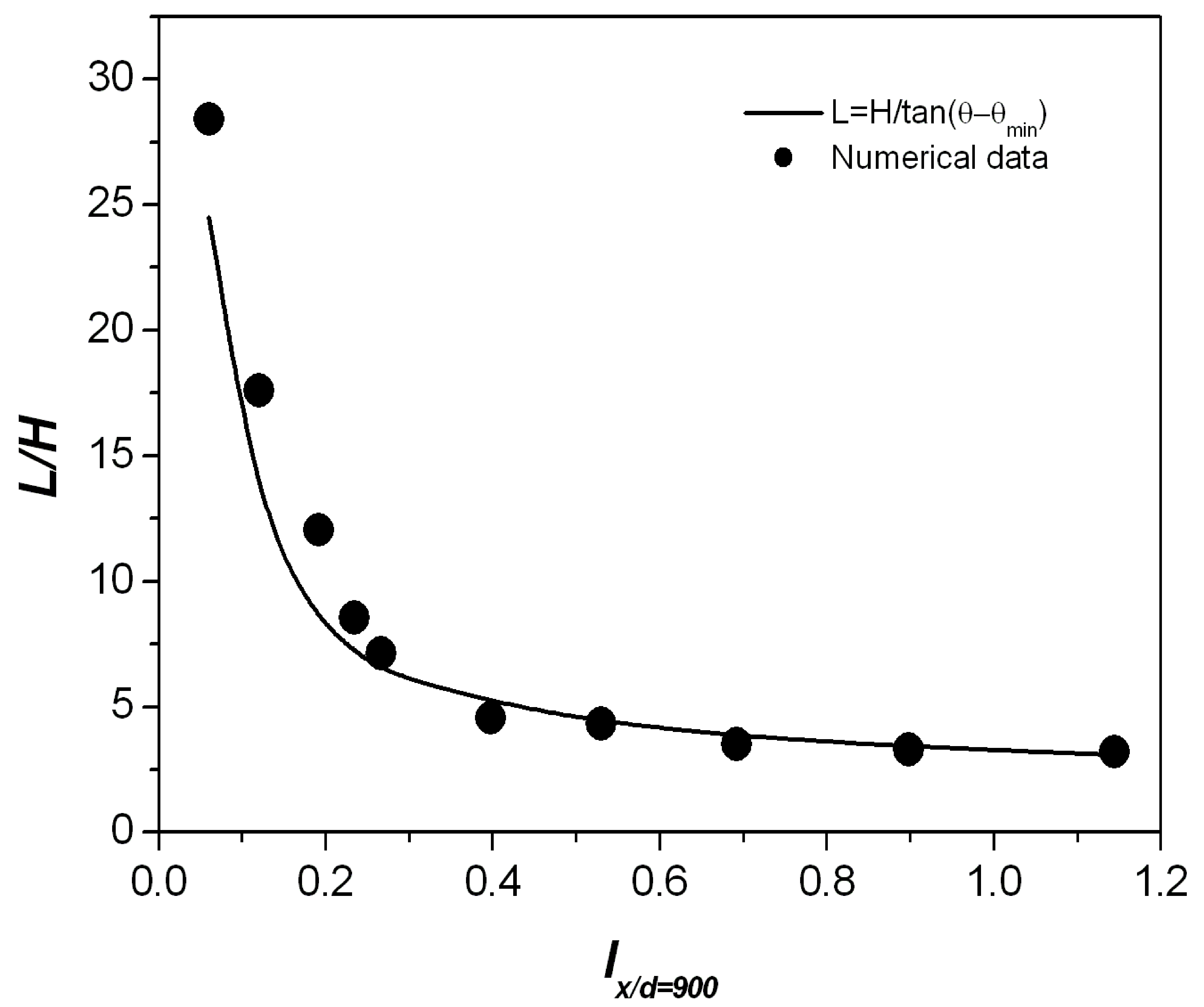




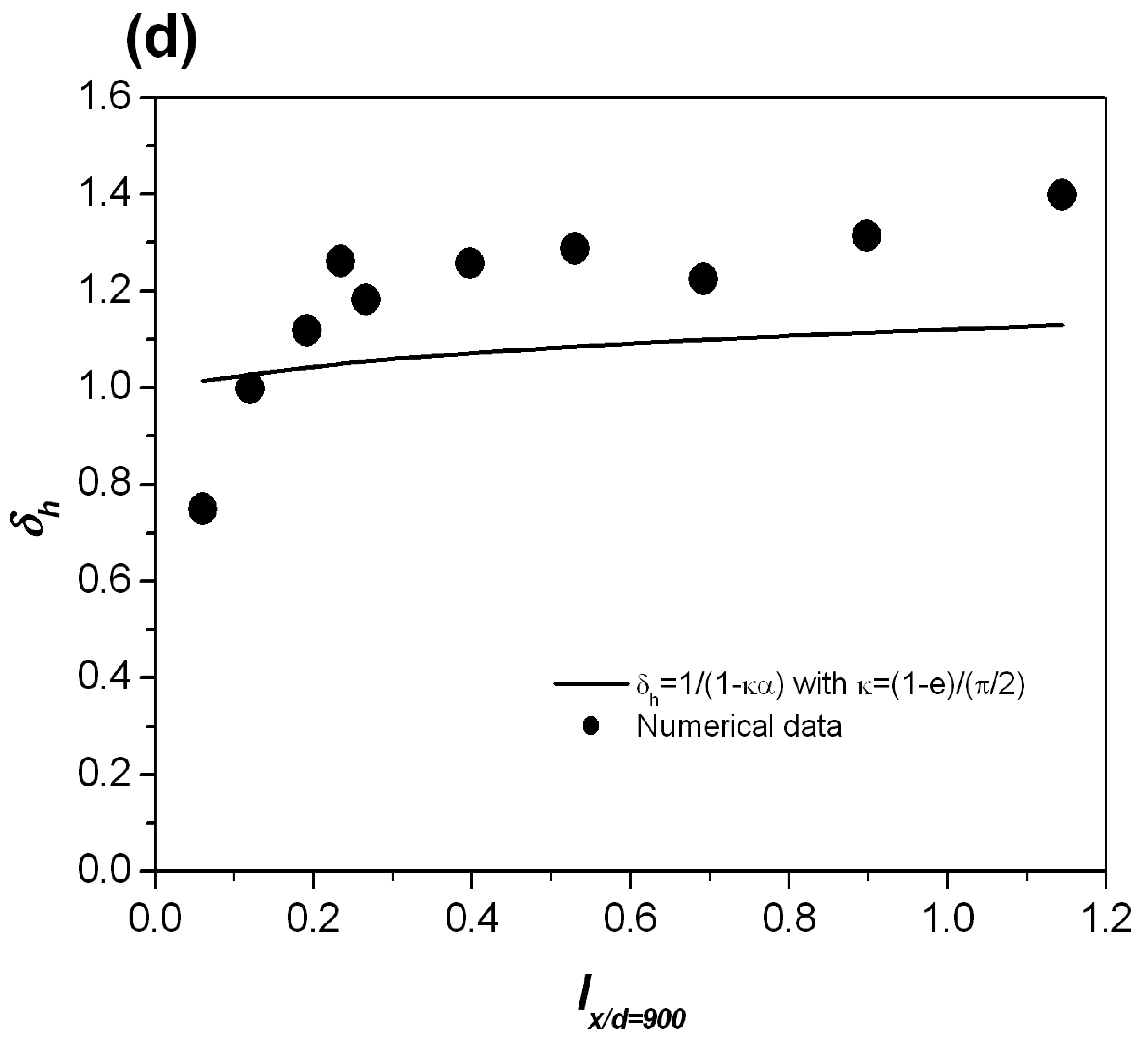




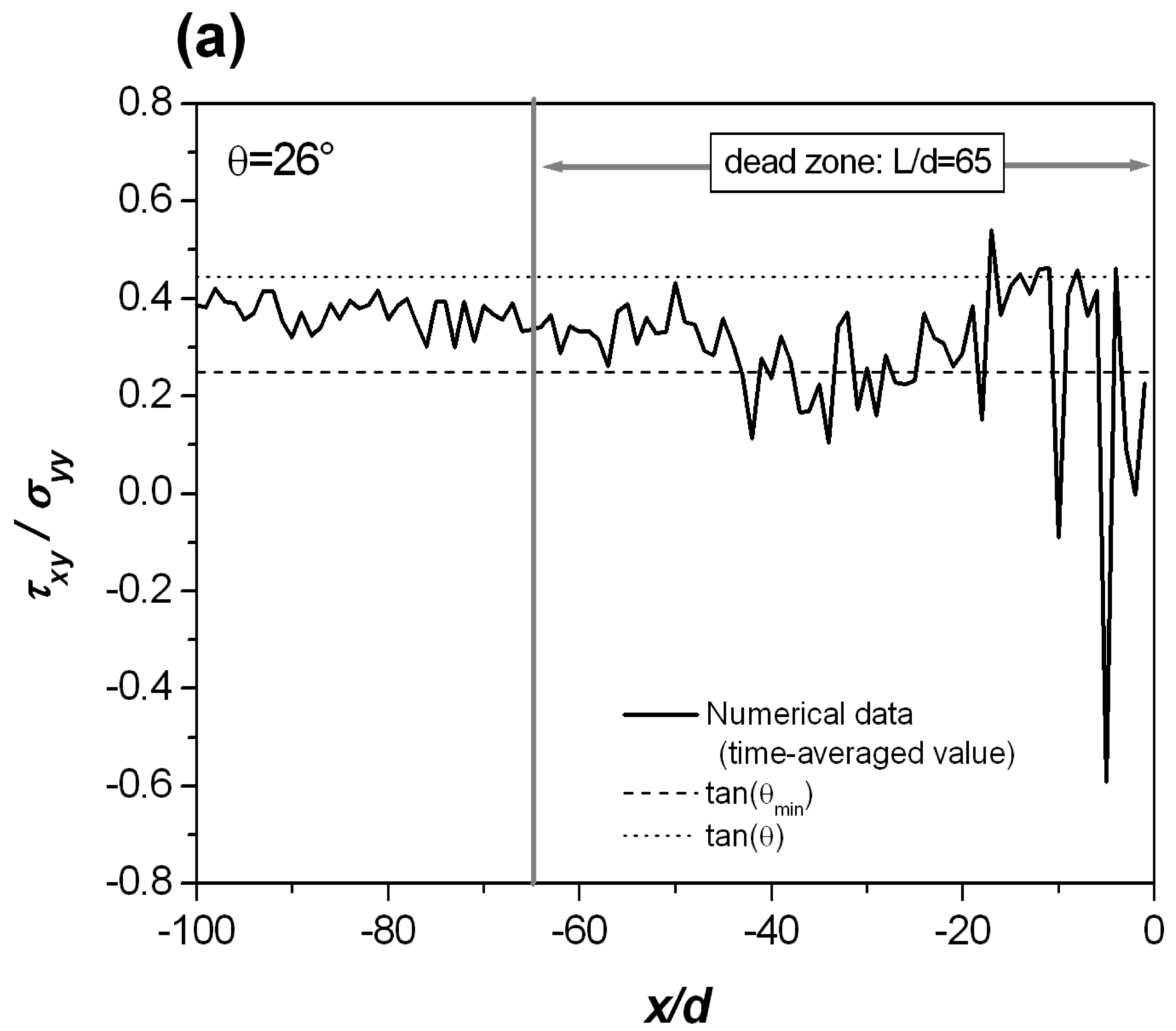


(b)

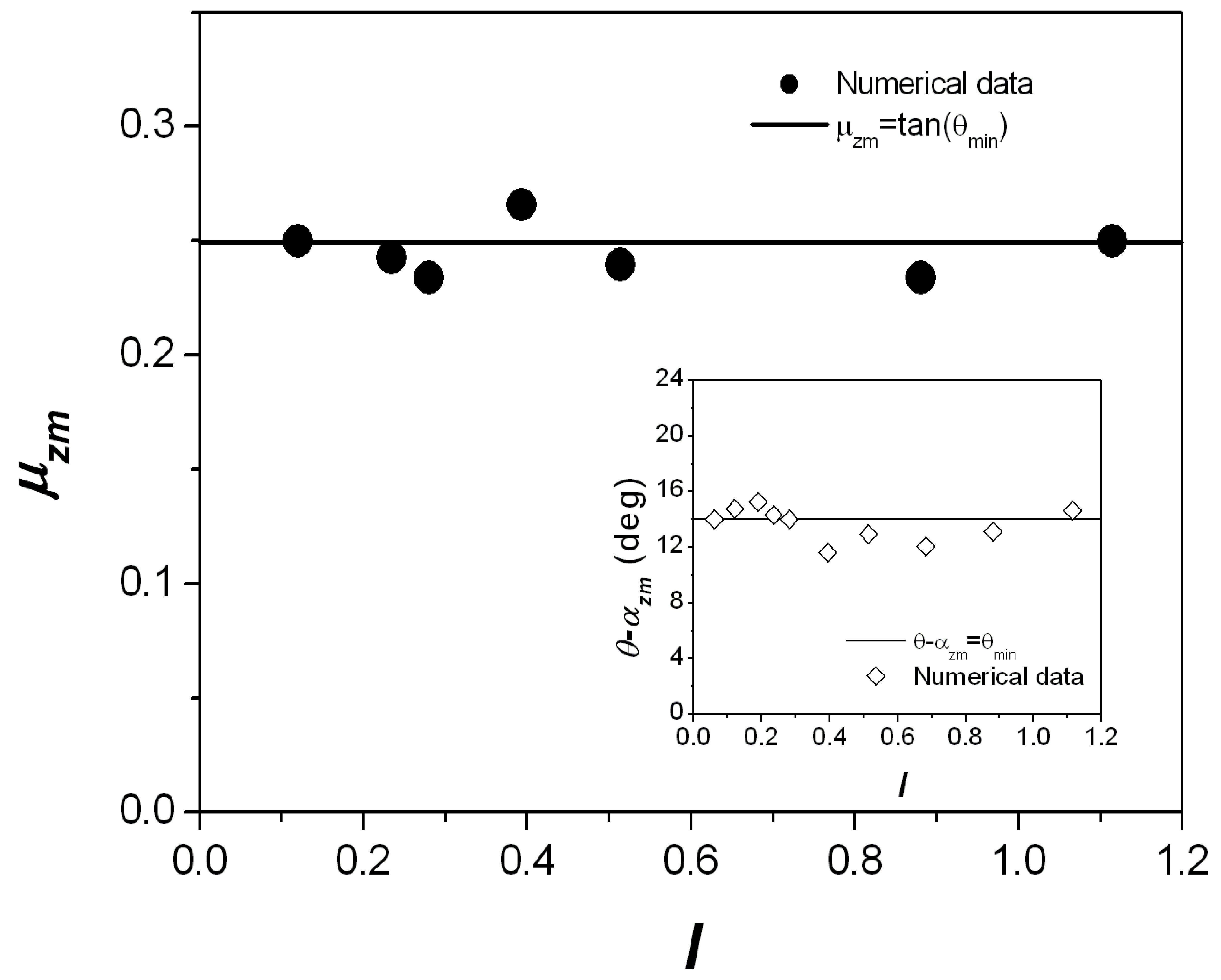




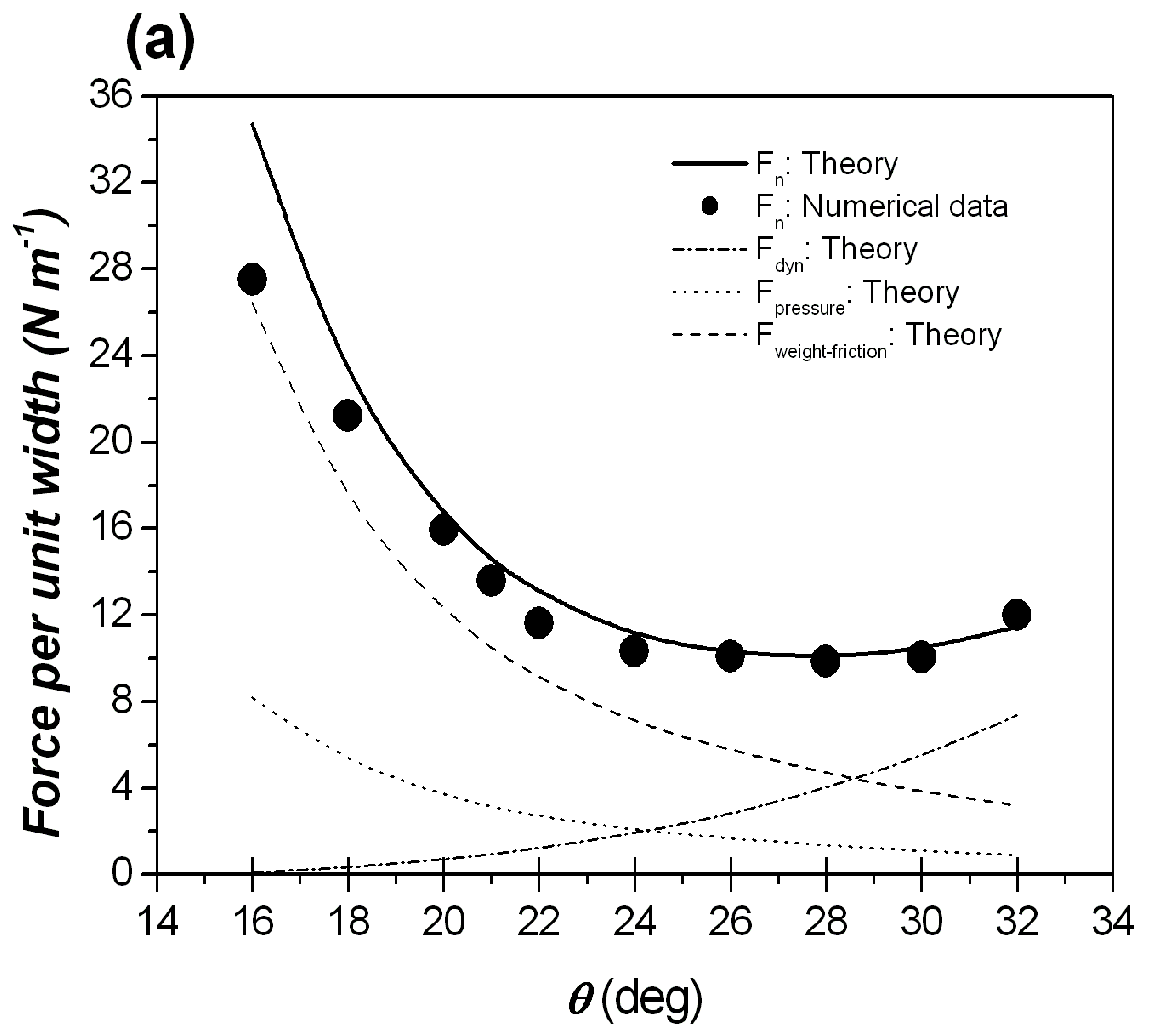




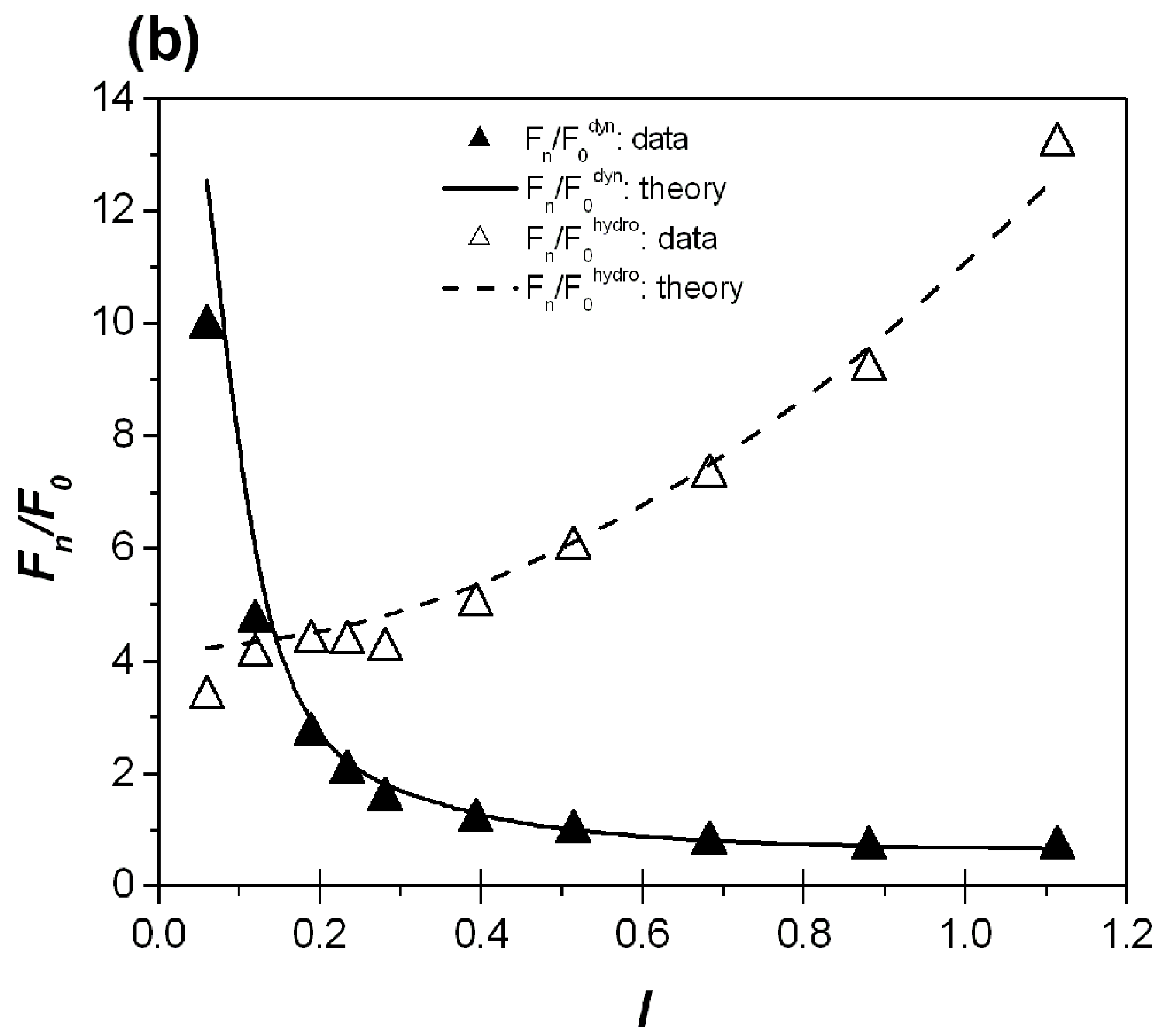




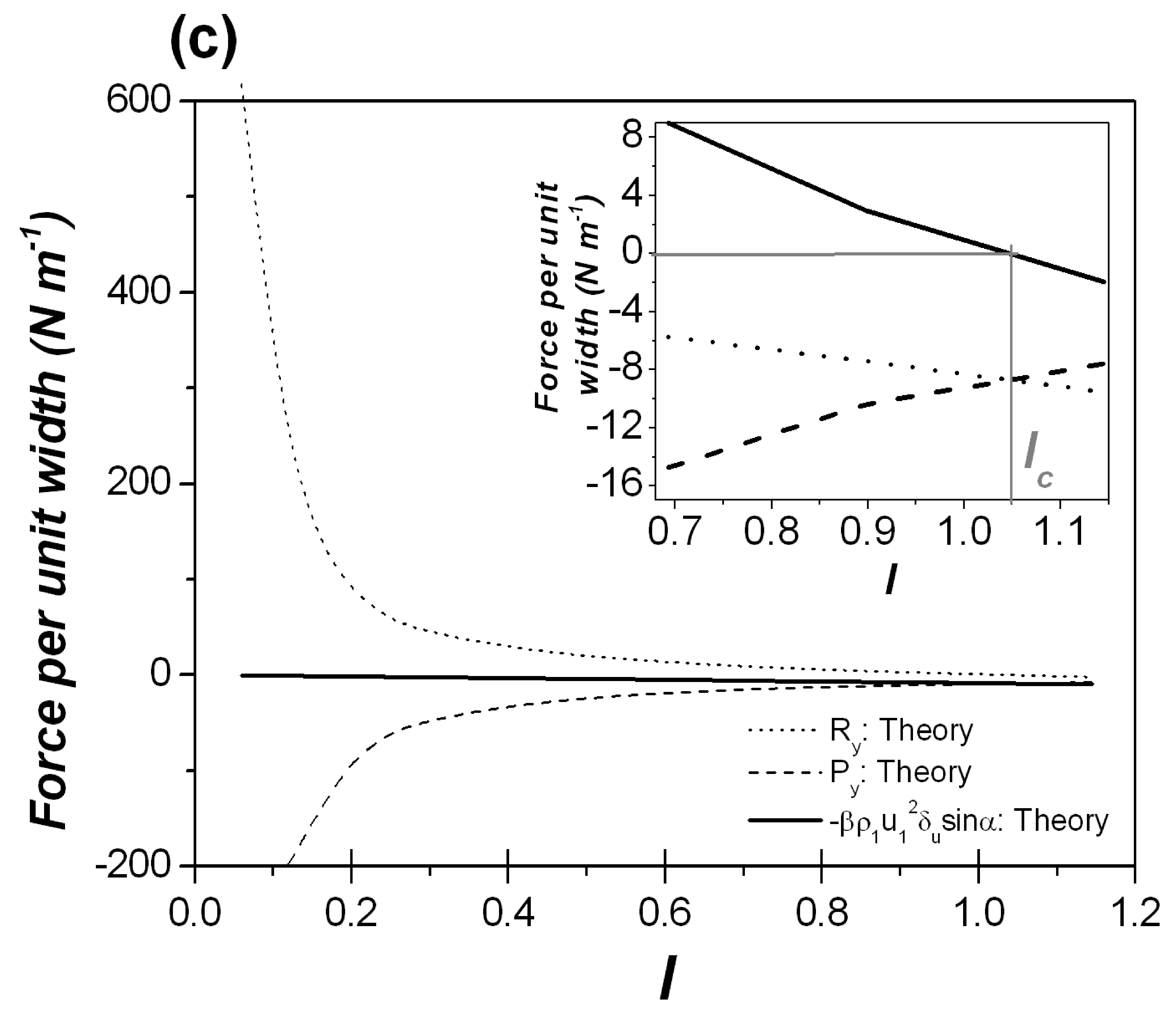

